DESY $01-023$

February 2001

hep-ph/0103005

\title{
Nonminimal Supersymmetric Standard Model with Baryon and Lepton Number Violation
}

\author{
P. N. Pandita \\ Theory Group, Deutsches Elektronen-Synchrotron DESY, D-22607 Hamburg, Germany \\ Department of Physics, North-Eastern Hill University, Shillong 793 022, India*
}

\begin{abstract}
We carry out a comprehensive analysis of the nonminimal supersymmetric standard model (NMSSM) with baryon and lepton number violation. We catalogue the baryon and lepton number violating dimension four and five operators of the model. We then study the renormalization group evolution and infrared stable fixed points of the Yukawa couplings and the soft supersymmetry breaking trilinear couplings of this model with baryon and lepton number (and R-parity) violation involving the heaviest generations. We show analytically that in the Yukawa sector of the NMSSM there is only one infrared stable fixed point. This corresponds to a non-trivial fixed point for the top-, bottom-quark Yukawa couplings and the $B$ violating coupling $\lambda_{233}^{\prime \prime}$, and a trivial one for all other couplings. All other possible fixed points are either unphysical or unstable in the infra-red region. We also carry out an analysis of the renormalization group equations for the soft supersymmetry breaking trilinear couplings, and determine the corresponding fixed points for these couplings. We then study the quasi-fixed point behaviour, both of the third generation Yukawa couplings and the baryon number violating coupling, and those of the soft supersymmetry breaking trilinear couplings. From the analysis of the fixed point behaviour, we obtain upper and lower bounds on the baryon number violating coupling $\lambda_{233}^{\prime \prime}$, as well as on the soft supersymmetry breaking trilinear couplings. Our analysis shows that the infrared fixed point behavior of NMSSM with baryon and lepton number violation is similar to that of MSSM.
\end{abstract}

PACS number(s): 11.10.Hi, 11.30.Fs, 12.60.Jv

*Permanent address 


\section{INTRODUCTION}

Supersymmetry [1] is at present the only known framework in which the Higgs sector of the Standard Model (SM), so crucial for its internal consistency, is natural. A much favored implementation of the idea of low energy supersymmetry is the Minimal Supersymmetric Standard Model (MSSM), obtained by simply doubling the number of states of the SM, and introducing a second Higgs doublet (with opposite hypercharge to the SM Higgs doublet) to generate masses for all the fermions and to cancel traingle gauge anomalies. The minimal supersymmetric version of the standard model leads to a successful prediction for the ratio of the gauge couplings with a gauge unification scale $M_{G} \simeq 10^{16} \mathrm{GeV}$. This has led to the idea that there may be a stage of unification beyond the SM. If so, then it becomes important to perform the radiative corrections in determining all the dimension $\leq 4$ terms in the lagrangian. This can be achieved by using the renormalization group equations in finding the values of parameters at the low scale, given their value at a high scale. Thus, considerable attention has recently been focussed on the renormalization group evolution [2] of the various dimensionless Yukawa couplings in the SM and its minimal supersymmetric extension, the Minimal Supersymmetric Standard Model. Using the renormalization group evolution, one may attempt to relate the Yukawa couplings to the gauge couplings via the Pendleton-Ross infra-red stable fixed point (IRSFP) for the top-quark Yukawa coupling [3], or via the quasifixed point behaviour [4]. The predictive power of the SM and its supersymmetric extensions may, thus, be enhanced if the renormalization group ( $R G$ ) running of the parameters is dominated by infra-red stable fixed points (IRSFPs). Typically, these fixed points are for ratios like Yukawa couplings to the gauge coupling, or in the context of supersymmetric models, the supersymmetry breaking trilinear $A$-parameter to the gaugino mass, etc. These ratios do not attain their fixed point values at the weak scale, the range between the GUT (or Planck) scale and the weak scale being too small for the ratios to closely approach the fixed point. Nevertheless, the couplings may be determined by quasi-fixed point behaviour [4 where the value of the Yukawa coupling at the weak scale is independent of its value at the GUT scale, provided the Yukawa coupling at the GUT scale is large. For the fixed point or the quasi-fixed point scenarios to be successful, it is necessary that these fixed points are stable [5].

Since supersymmetry [1] requires the introduction of superpartners of all known particles in the SM (in addition to the introduction of at least two Higgs doublets), which transform in an identical manner under the gauge group, there are additional Yukawa couplings in supersymmetric models which violate [8] baryon number $(B)$ or lepton number $(L)$. In MSSM, a discrete symmetry [9] called R-parity $\left(R_{p}\right)$ is invoked to eliminate these $B$ and $L$ violating Yukawa couplings. However, the assumption of $R_{p}$ conservation at the level of the MSSM appears to be ad hoc, since it is not required for the internal consistency of the model. Therefore, the study of the renormalization group evolution of the dimensionless Yukawa couplings in the MSSM, including $B$ and $L$ (and $R_{p}$ ) violation, deserves serious consideration. Recently considerable attention has been devoted to this question [10,11]. It has been shown that the only stable infrared fixed point of MSSM with baryon and lepton number violation is the one where the top-, bottom-quark Yukawa couplings and the $B$ violating coupling $\lambda_{233}^{\prime \prime}$ approach a non-trivial fixed point, and the $\tau$-Yukawa coupling approaches a trivial fixed point. All other possible fixed points are either unphysical or 
unstable in the infrared region.

It is well known that the minimal supersymmetric standard model suffers from the socalled $\mu$ problem associated with the bilinear term connecting the two Higgs doublet superfields in the superpotential. A simple solution to this problem is to postulate the existence of a Higgs singlet superfield, and to couple it to the two Higgs doublets in the superpotential via a dimensionless trilinear coupling. When the Higgs singlet obtains a vacuum expectation value, a bilinear term involving the two Higgs doublets is naturally generated [12]. However, this leads to additional trilinear superpotential couplings in the model, the so called nonminimal supersymmetric standard model (NMSSM). Furthermore, if we do not postulate $R_{p}$ conservation, then there is also an additional lepton number violating superpotential coupling in this model as compared to the MSSM with baryon and lepton number violation. Because of these additional trilinear couplings, it is important to study the infra-red fixed point structure of the NMSSM, and analyze the effect of these additional Yukawa couplings on the infrared behavior of the other Yukawa, and the baryon- and lepton-number violating couplings, and contrast it with the situation that obtains in the MSSM. Some preliminary studies of the NMSSM with $B$ and $L$ violation were carried out in [13. In this paper we carry out a detailed study of the renormalization group evolution of the Yukawa couplings of NMSSM, including the $B$ and $L$ violating couplings. We shall include all the third generation Yukawa couplings, as well as the highest generation $B$ and $L$ violating couplings in our study, and analyze the situation where all of them could simultaneously approach infrared fixed points. We shall investigate both the true infrared fixed points, as well as quasi-fixed-points of these couplings. In particular, we shall carry out a detailed stability analysis of the infrared fixed points of these couplings. Furthermore, corresponding to the $B$ and $L$ (and $R_{p}$ ) violating Yukawa couplings of the NMSSM, there are soft supersymmetry breaking trilinear couplings (the $A$ parameters) whose renormalization group evolution and infrared fixed point structure has not been studied so far. We shall, therefore, also study the renormalization group evolution of these soft supersymmetry breaking $A$ parameters, including those corresponding to the third generation Yukawa couplings, and obtain the simultaneous infrared fixed points for them.

The plan of the paper is as follows. In Sec. II we describe the model and write down all the dimension four and five baryon and lepton number violating couplings in the NMSSM. We then derive the renormalization group equations (RGE's) of interest to us, which include the equations for the dimension four baryon and lepton number violating Yukawa couplings. We then carry out a detailed analytical study of the true infrared fixed points of the Yukawa couplings in full generality. Within the context of grand unified theories, one is led to the situation where $B$ and $L$ violating Yukawa couplings may be related at the GUT scale, and one may no longer be able to set one or the other arbitarily to zero. We, therefore, initially include both baryon and lepton number violating couplings in our $R G$ equations. The fixed point analysis of such a system of RG equations leads to the crucial result that the only stable fixed point is the one with simultaneous non-trivial fixed point values for the topand bottom-quark Yukawa couplings and the $B$-violating coupling $\lambda_{233}^{\prime \prime}$, and a trivial one for all other couplings. Thus, non-trivial simultaneous fixed points for the $B$ and $L$ violating Yukawa couplings are ruled out by our analysis of the NMSSM. This is result is analogous to the corresponding result obtained in the MSSM [11]. We then study the fixed points of the corresponding soft supersymmetry breaking trilinear couplings of this model. In Sec. III we 
algebraically study the simultaneous quasi-fixed points of all the third generation Yukawa couplings of the minimal supersymmetric standard model with $B$ violation, as well as those of the corresponding soft supersymmetry breaking trilinear couplings. Since the quasi-fixed point limit is formally defined as the Landau pole of the Yukawa coupling at the GUT scale, it provides an upper bound on the corresponding Yukawa coupling. In Sec. IV we present the numerical results for the renormalization group evolution and the quasi-fixed points for the nonminimal supersymmetric standard model with $B$ violation. In Sec. V we summarize our results and present the conclusions.

\section{RENORMALIZATION GROUP EQUATIONS AND INFRA-RED FIXED POINTS}

\section{A. Baryon and lepton number violation in NMSSM}

In this section we study the true infra-red fixed points of the Yukawa couplings and the $A$ parameters of the NMSSM with $B$ and $L$ violation. We begin by recalling the basic features of the NMSSM with baryon and lepton number violation. The superpotential of the model is written as

$$
W=\left(h_{U}\right)_{a b} Q_{L}^{a} \bar{U}_{R}^{b} H_{2}+\left(h_{D}\right)_{a b} Q_{L}^{a} \bar{D}_{R}^{b} H_{1}+\left(h_{E}\right)_{a b} L_{L}^{a} \bar{E}_{R}^{b} H_{1}+\lambda N H_{1} H_{2}-\frac{\kappa}{3} N^{3} .
$$

where $L, Q, \bar{E}, \bar{D}, \bar{U}$ denote the lepton and quark doublets, and anti-lepon singlet, d-type anti-quark singlet and u-type anti-quark singlet, respectively. In Eq. (1), $\left(h_{U}\right)_{a b},\left(h_{D}\right)_{a b}$ and $\left(h_{E}\right)_{a b}$ are the Yukawa coupling matrices, with $a, b, c$ as the generation indices. Gauge invariance, supersymmetry and renormalizability allow the addition of the following $L$ and $B$ violating terms to the superpotential (1):

$$
\begin{aligned}
W_{L} & =\tilde{\lambda}_{a} N L_{a} H_{2}+\frac{1}{2} \lambda_{a b c} L_{L}^{a} L_{L}^{b} \bar{E}_{R}^{c}+\lambda_{a b c}^{\prime} L_{L}^{a} Q_{L}^{b} \bar{D}_{R}^{c}, \\
W_{B} & =\frac{1}{2} \lambda_{a b c}^{\prime \prime} \bar{D}_{R}^{a} \bar{D}_{R}^{b} \bar{U}_{R}^{c},
\end{aligned}
$$

where the notation 13 is standard. We note that there is a additional $L$-violating term with the dimensionless Yukawa coupling $\tilde{\lambda}_{a}$ in (2) which does not have an analogue in the MSSM. This term can be rotated away into the R-parity conserving term $\lambda \mathrm{NH}_{1} \mathrm{H}_{2}$ via an $S U(4)$ rotation between the superfields $H_{1}$ and $L_{a}$. However, this rotation must be performed at some energy scale, and the term is regenerated through the renormalization group equations. The Yukawa couplings $\lambda_{a b c}$ and $\lambda_{a b c}^{\prime \prime}$ are antisymmetric in their first two indices due to $S U(2)_{L}$ and $S U(3)_{C}$ group symmetries, respectively.

The dimension-4 terms in the supertentials (2) and (3) are the most dangerous terms for nucleon decay, and some of them must be suppressed. This leads to constraints [14] on the different couplings $\lambda_{a b c}, \lambda_{a b c}^{\prime}$, and $\lambda_{a b c}^{\prime \prime}$, but considerable freedom remains for the various $B$ and $L$ violating couplings. Furthermore, there are dimension- 5 operators which may lead to nucleon decay suppressed by $1 / M$, where $M$ is some large mass scale at which the $B$ and $L$ violation beyond that of NMSSM (and MSSM) comes into play. Some of these dimension-5 operators may also lead to unacceptable nucleon decay if their coeffcients are of order unity, 
and therefore must be suppressed. We tabulate here all the dimension-5 operators which are allowed by the $S U(3)_{C} \times S U(2)_{L} \times U(1)_{Y}$ gauge symmetry and the particle content of the NMSSM. These are

$$
\begin{array}{ll}
\mathcal{O}_{1}=[Q Q Q L]_{F}, & \mathcal{O}_{2}=[\bar{U} \bar{U} \bar{D} E]_{F}, \\
\mathcal{O}_{3}=\left[Q Q Q H_{1}\right]_{F}, & \mathcal{O}_{4}=\left[Q \bar{U} \bar{E} H_{1}\right]_{F}, \\
\mathcal{O}_{5}=\left[L L H_{2} H_{2}\right]_{F}, & \mathcal{O}_{6}=\left[L H_{1} H_{2} H_{2}\right]_{F}, \\
\mathcal{O}_{7}=\left[H_{2} H_{2} \bar{E}^{*}\right]_{D}, & \mathcal{O}_{8}=\left[H_{2}^{*} H_{1} \bar{E}\right]_{D}, \\
\mathcal{O}_{9}=\left[Q \bar{U} L^{*}\right]_{D}, & \mathcal{O}_{10}=\left[\bar{U} \bar{D}^{*} \bar{E}\right]_{D}, \\
\mathcal{O}_{11}=\left[L H_{2} N N\right]_{F}, & \mathcal{O}_{12}=[L L \bar{E} N]_{F}, \\
\mathcal{O}_{13}=[L Q \bar{D} N]_{F}, & \mathcal{O}_{14}=[\bar{D} \bar{D} \bar{U} N]_{F},
\end{array}
$$

where we have suppressed the gauge and family indices. We note that the baryon and lepton number violating operators $\mathcal{O}_{1}, \ldots \ldots \ldots, \mathcal{O}_{10}$ are the same as the corresponding operators in MSSM and are subject to constraints similar to those in MSSM, whereas the operators $\mathcal{O}_{11}, \ldots \ldots, \mathcal{O}_{15}$ are additional dimension-5 $B$ and $L$ violating operators specific to the NMSSM. We note that in NMSSM the simutaneous presence of the combinations $L Q \bar{D} N$ and $\bar{D} \bar{D} \bar{U}$, or $L Q \bar{D}$ and $\bar{D} \bar{D} \bar{U} N$ needs to be forbidden, since these could lead to fast proton decay. We do not consider the dimension-5 operators (田) any further in this paper, and restrict our attention only to the dimension-4 terms in (11), (2) and (3), respectively.

Corresponding to the terms in the superpotentials (1), (2) and (3), there are the soft supersymmetry breaking trilinear terms which can be written as

$$
\begin{aligned}
-V_{\text {soft }} & =\left[\left(A_{U}\right)_{a b}\left(h_{U}\right)_{a b} \tilde{Q}_{L}^{a} \tilde{\bar{U}}_{R}^{b} H_{2}+\left(A_{D}\right)_{a b}\left(h_{D}\right)_{a b} \tilde{Q}_{L}^{a} \tilde{\bar{D}}_{R}^{b} H_{1}\right. \\
& \left.+\left(A_{E}\right)_{a b}\left(h_{E}\right)_{a b} \tilde{L}_{L}^{a} \tilde{\bar{E}}_{R}^{b} H_{1}+A_{\lambda} \lambda N H_{1} H_{2}-\frac{A_{\kappa}}{3} \kappa N^{3}\right] \\
& +\left[\left(A_{\tilde{\lambda}}\right)_{a} \tilde{\lambda}_{a} N \tilde{L}_{L}^{a} H_{2}+\frac{1}{2}\left(A_{\lambda}\right)_{a b c} \lambda_{a b c} \tilde{L}_{L}^{a} \tilde{L}_{L}^{b} \tilde{\bar{E}}_{R}^{c}+\left(A_{\lambda^{\prime}}\right)_{a b c} \lambda_{a b c}^{\prime} \tilde{L}_{L}^{a} \tilde{Q}_{L}^{b} \tilde{\bar{D}}_{R}^{c}\right] \\
& +\left[\frac{1}{2}\left(A_{\lambda^{\prime \prime}}\right)_{a b c} \lambda_{a b c}^{\prime \prime} \tilde{\bar{D}}_{R}^{a} \tilde{\bar{D}}_{R}^{b} \tilde{\bar{U}}_{R}^{c}\right],
\end{aligned}
$$

where a tilde over a matter chiral superfield denotes its scalar component, and the notation for the scalar component of the Higgs superfield is the same as that of the corresponding superfield. In addition there are soft supersymmetry breaking gaugino mass terms with the masses $M_{i}$, with $i=1,2,3$ corresponding to the gauge groups $U(1)_{Y}, S U(2)_{L}$, and $S U(3)_{C}$, respectively.

The third generation Yukawa couplings are the dominant couplings in the superpotential (11). Therefore, it is natural to retain only the elements $\left(h_{U}\right)_{33} \equiv h_{t},\left(h_{D}\right)_{33} \equiv h_{b}, \quad\left(h_{L}\right)_{33} \equiv$ $h_{\tau}$ in each of the Yukawa couplings matrices $h_{U}, h_{D}, h_{L}$, setting all other elements equal to zero. Furthermore, there are 39 independent $L$ violating trilinear couplings $\tilde{\lambda}_{a}, \lambda_{a b c}$ and $\lambda_{a b c}^{\prime}$ in (2). Similarly, there are 9 independent $B$ violating couplings $\lambda_{a b c}^{\prime \prime}$ in the baryon number violating superpotential (3). Thus, we would have to consider 44 coupled nonlinear evolution equations for the $L$ violating case and 14 coupled nonlinear equations for the $B$ 
violating case, respectively to study the renormalization group evolution of these couplings in the NMSSM. It is clear that there is a need for a radical simplification of these equations before we can think of studying the evolution of the Yukawa couplings in the NMSSM with $B$ and $L$ violation.

In order to render the Yukawa couping evolution equations tractable, we, therefore, need to make certain plausible assumptions. Motivated by the generational hierarchy of the conventional Yukawa couplings, we shall assume that an analogous hierarchy amongst the different generations of $B$ and $L$ violating couplings exists. Thus, we shall retain only the couplings $\tilde{\lambda}_{3}, \lambda_{233}, \quad \lambda_{333}^{\prime}, \lambda_{233}^{\prime \prime}$, and neglect the rest. We note that $B$ and $L$ violating couplings to higher generations evolve more strongly because of larger Higgs couplings in their evolution equations, and hence could take larger values than the corresponding couplings to the lighter generations. We also note that the experimental upper limits are stronger for the $B$ and $L$ violating couplings in MSSM with lower indices [14].

\section{B. Renormalization group equations}

We are interested in the one-loop renormalization group equations for the dimensionless trilinear Yukawa couplings in the superpotential (17), (22) and (3)). For a general $\mathrm{N}=1$ supersymmetric theory with a trilinear superpotential term $f_{a b c} \Phi^{a} \Phi^{b} \Phi^{c}$ involving chiral superfields $\Phi^{a}, \Phi^{b}, \Phi^{c}$, the evolution of the couplings $f_{a b c}$ with the scale parameter $\mu$ is given by the RGEs [15]

$$
16 \pi^{2} \frac{\partial f_{a b c}}{\partial \ln \mu}=\gamma_{a}^{e} f_{e b c}+\gamma_{b}^{e} f_{a e c}+\gamma_{c}^{e} f_{a b e},
$$

where $\gamma_{a}^{e}$ are the elements of the anomalous dimension matrix, and sum over repeated indices is understood. The anomalous dimensions are given by

$$
\gamma_{a}^{e}=\frac{1}{2} \sum_{b, c} f_{a b c} f^{e b c}-2 \delta_{a}^{e} g_{A}^{2} C_{a}^{A},
$$

to one loop order. The sum over A represents a sum over all dominant gauge couplings, and $C_{a}^{A}$ is the quadratic Casimir of the representation of $\Phi^{a}$ under the gauge group with coupling $g_{A}$ :

$$
\left(T_{R}^{A} T_{R}^{A}\right)_{a}^{b}=C_{R}^{A} \delta_{a}^{b} .
$$

Here, $T_{R}^{A}$ is a matrix in the $\mathrm{R}$ representation for the group labelled by A. Pictorially, the $\mathrm{RG}$ evolution of the trilinear coupling can be described as an insertion of the anomalous dimension correction on each external leg. We have calculated the anomalous dimension for the various superfields for the NMSSM with baryon- and lepton-number violating couplings.

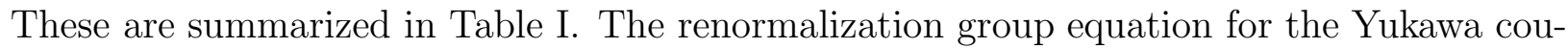
plings $h_{U}, h_{D}, h_{E}$ of the superpotential (11) are obtained from (6) with the index c belonging to a Higgs field. The general form of the RGEs are 


\begin{tabular}{|lccc|}
\hline \hline \multicolumn{1}{|c}{$\Phi^{a, b}$} & NMSSM & L violation & B violation \\
\hline$\hat{N}, \hat{N}$ & $4 \lambda^{2}+4 k^{2}$ & $\lambda^{i} \lambda_{i}$ & - \\
$\hat{L}_{i}, \hat{H}_{1}$ & - & $\lambda^{i a b}\left(h_{E}\right)_{a b}+3 \lambda^{\prime i a b}\left(h_{D}\right)_{a b}+\lambda \lambda^{i}$ & - \\
$\hat{L}_{i, j}$ & $h_{L} h_{L}^{\dagger}-\frac{3}{2} g_{2}^{2}-\frac{3}{10} g_{1}^{2}$ & $\lambda_{i a b} \lambda^{j a b}+3 \lambda_{i a \lambda^{\prime}}^{\prime} \lambda^{\prime j a b}+\lambda_{i} \lambda^{i} \delta_{j}^{i}$ & - \\
$\hat{E}_{i, j}^{c}$ & $2 h_{E}^{\dagger} h_{E}-\frac{6}{5} g_{1}^{2}$ & $\lambda^{a b i} \lambda_{a b j}$ & - \\
$\hat{D}_{i, j}^{c}$ & $2 h_{D}^{\dagger} h_{D}-\frac{8}{3} g_{3}^{2}-\frac{2}{15} g_{1}^{2}$ & $2 \lambda^{\prime a b i} \lambda_{a b j}^{\prime}$ & $2 \lambda^{\prime \prime i a b} \lambda_{j a b}^{\prime \prime}$ \\
$\hat{U}_{i, j}^{c}$ & $2 h_{U}^{\dagger} h_{U}-\frac{8}{3} g_{3}^{2}-\frac{8}{15} g_{1}^{2}$ & - & $\lambda^{\prime \prime a b i} \lambda_{a b j}^{\prime \prime}$ \\
$\hat{Q}_{i, j}$ & $h_{U} h_{U}^{\dagger}+h_{D} h_{D}^{\dagger}-\frac{8}{3} g_{3}^{2}-\frac{3}{2} g_{2}^{2}-\frac{1}{30} g_{1}^{2}$ & $\lambda_{a i b}^{\prime} \lambda^{\prime a j b}$ & - \\
$\hat{H}_{1}, \hat{H}_{1}$ & $\operatorname{Tr}\left(h_{E} h_{E}^{\dagger}+3 h_{D} h_{D}^{\dagger}\right)+\lambda^{2}-\frac{3}{2} g_{2}^{2}-\frac{3}{10} g_{1}^{2}$ & - & - \\
$\hat{H}_{2}, \hat{H}_{2}$ & $3 \operatorname{Tr}\left(h_{U} h_{U}^{\dagger}\right)+\lambda^{2}-\frac{3}{2} g_{2}^{2}-\frac{3}{10} g_{1}^{2}$ & $\lambda_{i} \lambda^{i}$ & - \\
\hline \hline
\end{tabular}

TABLE I. The anomalous dimensions $\gamma_{\Phi^{b}}^{\Phi^{a}}$ in the non-minimal supersymmetric standard model with lepton and baryon number violating couplings. Here $a, b$ are flavour indices.

$$
\begin{aligned}
16 \pi^{2} \frac{\partial}{\partial \ln \mu}\left(h_{U}\right)_{a b} & =\left(h_{U}\right)_{i b} \gamma_{Q_{a}}^{Q_{i}}+\left(h_{U}\right)_{a i} \gamma_{\bar{U}_{b}}^{\bar{U}_{i}}+\left(h_{U}\right)_{a b} \gamma_{H_{2}}^{H_{2}}, \\
16 \pi^{2} \frac{\partial}{\partial \ln \mu}\left(h_{D}\right)_{a b} & =\left(h_{D}\right)_{i b} \gamma_{Q_{a}}^{Q_{i}}+\left(h_{D}\right)_{a i} \gamma_{\bar{D}_{i}}^{\bar{D}_{i}}+(D)_{a b} \gamma_{H_{1}}^{H_{1}}+\lambda_{i a b}^{\prime} \gamma_{H_{1}}^{L_{i}}, \\
16 \pi^{2} \frac{\partial}{\partial \ln \mu}\left(h_{L}\right)_{a b} & =\left(h_{L}\right)_{i b} \gamma_{L_{a}}^{L_{i}}+\left(h_{L}\right)_{a i} \gamma_{\bar{E}_{b}}^{\bar{E}_{i}}+\left(h_{L}\right)_{a b} \gamma_{H_{1}}^{H_{1}}+\lambda_{i a b} \gamma_{H_{1}}^{L_{i}} .
\end{aligned}
$$

The evolution of gauge couplings $g_{i}\left(i=1,2,3\right.$ denoting the $U(1)_{Y}, S U(2)_{L}$ and $S U(3)_{C}$ gauge groups, with GUT normalization for $U(1)_{Y}$ gauge group) in NMSSM with $B$ - and $L$-violation is same as in MSSM, as this evolution is unaffected at the one-loop level by the presence of a chiral singlet superfield, or B- and L-violating couplings. These evolution equations are

$$
16 \pi^{2} \frac{d g_{i}}{d \ln \mu}=b_{i} g_{i}^{3}, \quad i=1,2,3
$$

where $b_{i}$ are the beta functions for the respective gauge couplings with $b_{1}=33 / 5, b_{2}=$ $1, b_{3}=-3$. The corresponding one-loop renormalization group equations for the gaugino masses $M_{i}, \mathrm{i}=1,2,3$ can be written as

$$
16 \pi^{2} \frac{d M_{i}}{d \ln \mu}=2 g_{i}^{2} b_{i} M_{i}, \quad i=1,2,3 .
$$

Retaining only the third generation Yukawa couplings and the highest generation baryon and lepton number violating couplings, the RGEs for the Yukawa couplings and the R-parity violating couplings in the NMSSM can be written as 16

$$
\frac{d h_{t}}{d \ln \mu}=\frac{h_{t}}{16 \pi^{2}}\left[6 h_{t}^{2}+h_{b}^{2}+\lambda^{2}+\tilde{\lambda}_{3}^{2}+\lambda_{333}^{\prime 2}+2 \lambda_{233}^{\prime \prime 2}\right.
$$




$$
\begin{aligned}
& \left.-\left(\frac{16}{3} g_{3}^{2}+3 g_{2}^{2}+\frac{13}{15} g_{1}^{2}\right)\right] \\
& \frac{d h_{b}}{d \ln \mu}=\frac{1}{16 \pi^{2}}\left[\left(h_{t}^{2}+6 h_{b}^{2}+h_{\tau}^{2}+\lambda^{2}+6 \lambda_{333}^{\prime 2}+2 \lambda_{233}^{\prime \prime 2}\right) h_{b}+\lambda \tilde{\lambda}_{3} \lambda_{333}^{\prime}\right. \\
& \left.-\left(\frac{16}{3} g_{3}^{2}+3 g_{2}^{2}+\frac{7}{15} g_{1}^{2}\right) h_{b}\right] \\
& \frac{d h_{\tau}}{d \ln \mu}=\frac{h_{\tau}}{16 \pi^{2}}\left[3 h_{b}^{2}+4 h_{\tau}^{2}+\lambda^{2}+\tilde{\lambda}_{3}^{2}+4 \lambda_{233}^{2}+3 \lambda_{333}^{\prime 2}\right. \\
& \left.-\left(3 g_{2}^{2}+\frac{9}{5} g_{1}^{2}\right)\right] \\
& \frac{d \lambda}{d \ln \mu}=\frac{1}{16 \pi^{2}}\left[\left(3 h_{t}^{2}+3 h_{b}^{2}+h_{\tau}^{2}+4 \lambda^{2}+2 \kappa^{2}+4 \tilde{\lambda}_{3}^{2}\right) \lambda+3 h_{b} \tilde{\lambda}_{3} \lambda_{333}^{\prime}\right. \\
& \left.-\left(3 g_{2}^{2}+\frac{3}{5} g_{1}^{2}\right) \lambda\right] \\
& \frac{d \kappa}{d \ln \mu}=\frac{\kappa}{16 \pi^{2}}\left[6 \lambda^{2}+6 \kappa^{2}+6 \tilde{\lambda}_{3}^{2}\right] \text {, } \\
& \frac{d \tilde{\lambda}_{3}}{d \ln \mu}=\frac{1}{16 \pi^{2}}\left[\left(3 h_{t}^{2}+h_{\tau}^{2}+4 \lambda^{2}+2 \kappa^{2}+4 \tilde{\lambda}_{3}^{2}+\lambda_{233}^{2}+3 \lambda_{333}^{\prime 2}\right) \tilde{\lambda}_{3}\right. \\
& \left.+3 h_{b} \lambda \lambda_{333}^{\prime}-\left(3 g_{2}^{2}+\frac{3}{5} g_{1}^{2}\right) \tilde{\lambda}_{3}\right] \\
& \frac{d \lambda_{233}}{d \ln \mu}=\frac{\lambda_{233}}{16 \pi^{2}}\left[4 h_{\tau}^{2}+\tilde{\lambda}_{3}^{2}+4 \lambda_{233}^{2}+3 \lambda_{333}^{\prime 2}-\left(3 g_{2}^{2}+\frac{9}{5} g_{1}^{2}\right)\right], \\
& \frac{d \lambda_{333}^{\prime}}{d \ln \mu}=\frac{1}{16 \pi^{2}}\left[\left(h_{t}^{2}+6 h_{b}^{2}+h_{\tau}^{2}+\tilde{\lambda}_{3}^{2}+\lambda_{233}^{2}+6 \lambda_{333}^{\prime 2}+2 \lambda_{233}^{\prime \prime}\right) \lambda_{333}^{\prime}\right. \\
& \left.+h_{b} \lambda \tilde{\lambda}_{3}-\left(\frac{16}{3} g_{3}^{2}+3 g_{2}^{2}+\frac{7}{15} g_{1}^{2}\right) \lambda_{333}^{\prime}\right] \\
& \frac{d \lambda_{233}^{\prime \prime}}{d \ln \mu}=\frac{\lambda_{233}^{\prime \prime}}{16 \pi^{2}}\left[\left(2 h_{t}^{2}+2 h_{b}^{2}+2 \lambda_{333}^{\prime 2}+6 \lambda_{233}^{\prime \prime 2}\right)-\left(8 g_{3}^{2}+\frac{4}{5} g_{1}^{2}\right)\right] \text {. }
\end{aligned}
$$

We note that since the difference between the one-loop and two-loop results [17] for the infrared fixed points in MSSM is less than 10\%, we shall use one-loop renormalization group equations in the study of infrared fixed points in the NMSSM in this paper.

We now come to the evolution equations for the soft supersymmetry breaking trilinear parameters in the potential (5). The one-loop RGEs for these parameters can be deduced from the general expressions in ref. [18]. In this paper we shall assume the same kind of generational hierarchy for these trilinear parameters as was assumed for the corresponding Yukawa couplings. Thus, we shall consider, besides $A_{\lambda}$ and $A_{\kappa}$, only the highest generation trilinear coulings $\left(A_{U}\right)_{33} \equiv A_{t},\left(A_{D}\right)_{33} \equiv A_{b},\left(A_{L}\right)_{33} \equiv A_{\tau},\left(A_{\tilde{\lambda}}\right)_{3} \equiv A_{\tilde{\lambda}_{3}}\left(A_{\lambda}\right)_{233} \equiv A_{\lambda_{233}}$, $\left(A_{\lambda^{\prime}}\right)_{333} \equiv A_{\lambda_{333}^{\prime}},\left(A_{\lambda^{\prime \prime}}\right)_{233} \equiv A_{\lambda_{233}^{\prime \prime}}$, setting all other elements equal to zero. With this assumption the RGEs for the soft supersymmetry breaking trilinear parameters can be written as

$$
\frac{d A_{t}}{d \ln \mu}=\frac{1}{8 \pi^{2}}\left(6 A_{t} h_{t}^{2}+A_{b} h_{b}^{2}+A_{\lambda} \lambda^{2}+A_{\tilde{\lambda}_{3}} \tilde{\lambda}_{3}^{2}+A_{\lambda_{333}^{\prime}} \lambda_{333}^{\prime 2}+2 A_{\lambda_{233}^{\prime \prime}} \lambda_{233}^{\prime \prime 2}\right.
$$




$$
\begin{aligned}
& \left.-\frac{16}{3} M_{3} g_{3}^{2}-3 M_{2} g_{2}^{2}-\frac{13}{15} M_{1} g_{1}^{2}\right) \\
& \frac{d A_{b}}{d \ln \mu}=\frac{1}{8 \pi^{2}}\left(A_{t} h_{t}^{2}+6 A_{b} h_{b}^{2}+A_{\tau} h_{\tau}^{2}+A_{\lambda} \lambda^{2}+\frac{3}{2} A_{b} \lambda_{333}^{\prime 2}+\frac{9}{2} A_{\lambda_{333}^{\prime}} \lambda_{333}^{\prime 2}\right. \\
& +2 A_{\lambda_{233}^{\prime \prime}} \lambda_{233}^{\prime \prime 2}-\frac{A_{b} \lambda \tilde{\lambda}_{3} \lambda_{333}^{\prime}}{2 h_{b}}+\frac{A_{\lambda} \lambda \tilde{\lambda}_{3} \lambda_{333}^{\prime}}{h_{b}}+\frac{A_{\lambda_{333}^{\prime}} \lambda \tilde{\lambda}_{3} \lambda_{333}^{\prime}}{2 h_{b}} \\
& \left.-\frac{16}{3} M_{3} g_{3}^{2}-3 M_{2} g_{2}^{2}-\frac{7}{15} M_{1} g_{1}^{2}\right) \\
& \frac{d A_{\tau}}{d \ln \mu}=\frac{1}{8 \pi^{2}}\left(3 A_{b} h_{b}^{2}+4 A_{\tau} h_{\tau}^{2}+A_{\lambda} \lambda^{2}+A_{\tilde{\lambda}_{3}} \tilde{\lambda}_{3}^{2}+\frac{A_{\tau} \lambda_{233}^{2}}{2}+\frac{7 A_{\lambda_{233}} \lambda_{233}^{2}}{2}\right. \\
& \left.+3 A_{\lambda_{333}^{\prime}} \lambda_{333}^{\prime 2}-3 M_{2} g_{2}^{2}-\frac{9}{5} M_{1} g_{1}^{2}\right) \\
& \frac{d A_{\lambda}}{d \ln \mu}=\frac{1}{8 \pi^{2}}\left(3 A_{t} h_{t}^{2}+3 A_{b} h_{b}^{2}+A_{\tau} h_{\tau}^{2}+4 A_{\lambda} \lambda^{2}+\frac{A_{\lambda} \tilde{\lambda}_{3}^{2}}{2}+2 A_{\kappa} \kappa^{2}\right. \\
& +\frac{7 A_{\tilde{\lambda}_{3}} \tilde{\lambda}_{3}^{2}}{2}+\frac{3 A_{b} h_{b} \tilde{\lambda}_{3} \lambda_{333}^{\prime}}{\lambda}-\frac{3 A_{\lambda} h_{b} \tilde{\lambda}_{3} \lambda_{333}^{\prime}}{2 \lambda}+\frac{3 A_{\tilde{\lambda}_{3}} h_{b} \tilde{\lambda}_{3} \lambda_{333}^{\prime}}{2 \lambda} \\
& \left.-3 M_{2} g_{2}^{2}-\frac{3}{5} M_{1} g_{1}^{2}\right) \\
& \frac{d A_{\kappa}}{d \ln \mu}=\frac{6}{8 \pi^{2}}\left(A_{\lambda} \lambda^{2}+A_{\kappa} \kappa^{2}+A_{\tilde{\lambda}_{3}} \tilde{\lambda}_{3}^{2}\right), \\
& \frac{d A_{\tilde{\lambda}_{3}}}{d \ln \mu}=\frac{1}{8 \pi^{2}}\left(3 A_{t} h_{t}^{2}+A_{\tau} h_{\tau}^{2}+\frac{7 A_{\lambda} \lambda^{2}}{2}+2 A_{\kappa} \kappa^{2}+\frac{A_{\tilde{\lambda}_{3}} \lambda^{2}}{2}+4 A_{\tilde{\lambda}_{3}} \tilde{\lambda}_{3}^{2}\right. \\
& +A_{\lambda_{233}} \lambda_{233}^{2}+3 A_{\lambda_{333}^{\prime}} \lambda_{333}^{\prime 2}+\frac{3 A_{\lambda} h_{b} \lambda \lambda_{333}^{\prime}}{2 \tilde{\lambda}_{3}}-\frac{3 A_{\tilde{\lambda}_{3}} h_{b} \lambda \lambda_{333}^{\prime}}{2 \tilde{\lambda}_{3}} \\
& \left.+\frac{3 A_{\lambda_{333}^{\prime}} h_{b} \lambda \lambda_{333}^{\prime}}{\tilde{\lambda}_{3}}-3 M_{2} g_{2}^{2}-\frac{3}{5} M_{1} g_{1}^{2}\right) \\
& \frac{d A_{\lambda_{233}}}{d \ln \mu}=\frac{1}{8 \pi^{2}}\left(\frac{7 A_{\tau} h_{\tau}^{2}}{2}+A_{\tilde{\lambda}_{3}} \tilde{\lambda}_{3}^{2}+\frac{A_{\lambda_{233}} h_{\tau}^{2}}{2}+4 A_{\lambda_{233}} \lambda_{233}^{2}+3 A_{\lambda_{233}^{\prime}} \lambda_{333}^{\prime 2}\right. \\
& \left.-3 M_{2} g_{2}^{2}-\frac{9}{5} M_{1} g_{1}^{2}\right) \\
& \frac{d A_{\lambda_{333}^{\prime}}}{d \ln \mu}=\frac{1}{8 \pi^{2}}\left(A_{t} h_{t}^{2}+\frac{9 A_{b} h_{b}^{2}}{2}+A_{\tau} h_{\tau}^{2}+A_{\tilde{\lambda}_{3}} \tilde{\lambda}_{3}^{2}+A_{\lambda_{233}} \lambda_{233}^{2}\right. \\
& +\frac{3 A_{\lambda_{333}^{\prime}} h_{b}^{2}}{2}+6 A_{\lambda_{333}^{\prime}} \lambda_{333}^{\prime 2}+2 A_{\lambda_{233}^{\prime \prime}} \lambda_{233}^{\prime \prime 2}+\frac{A_{b} h_{b} \lambda \tilde{\lambda}_{3}}{2 \lambda_{333}^{\prime}}+\frac{A_{\tilde{\lambda}_{3}} h_{b} \lambda \tilde{\lambda}_{3}}{\lambda_{333}^{\prime}} \\
& \left.-\frac{A_{\lambda_{333}^{\prime}} h_{b} \lambda \tilde{\lambda}_{3}}{2 \lambda_{333}^{\prime}}-\frac{16}{3} M_{3} g_{3}^{2}-3 M_{2} g_{2}^{2}-\frac{7}{15} M_{1} g_{1}^{2}\right) \\
& \frac{d A_{\lambda_{233}^{\prime \prime}}}{d \ln \mu}=\frac{1}{8 \pi^{2}}\left(2 A_{t} h_{t}^{2}+2 A_{b} h_{b}^{2}+2 A_{\lambda^{\prime}} \lambda_{333}^{\prime 2}+6 A_{\lambda_{233}^{\prime \prime}} \lambda_{233}^{\prime \prime 2}\right.
\end{aligned}
$$




$$
\left.-8 M_{3} g_{3}^{2}-\frac{4}{5} M_{1} g_{1}^{2}\right) .
$$

Given the evolution equations (14) - (22) for the Yukawa couplings and the evolution equations (23) - (31) for the $A$ parameters, we are now ready to study the RG evolution and infra-red fixed points of the NMSSM with $B$ and $L$ violation.

\section{Infrared fixed points for Yukawa couplings}

In this section we consider the infrared fixed points for the Yukawa couplings and the baryon and lepton number violating couplings of the NMSSM. The infra-red fixed points [13] of the renormalization group equations (14) - (22) have been studied in the limit of ignoring the $\tau$-Yukawa coupling $h_{\tau}$, and by considering either the baryon number violating Yukawa coupling $\lambda_{233}^{\prime \prime}$, or the lepton number violating Yukawa couplings $\tilde{\lambda}_{3}, \lambda_{233}$ and $\lambda_{333}^{\prime}$. In the analysis that follows, we shall consider the evolution equations for $h_{t}$ and $h_{b}$ together with the evolution equation for $h_{\tau}$. Furthermore, we shall also entertain the possibility of simultaneous presence of $B$ and $L$ violating couplings in the renormalization group equations (14) - (22). We do this in order to investigate as to whether such a system of equations does have acceptable infrared fixed points. However, without loss of generality, we shall assume that there is a hierarchy of lepton number violating couplings, and consider only one lepton number violating coupling, together with the baryon number violating coupling $\lambda_{233}^{\prime \prime}$, at a time. Thus, we shall consider three different cases, i.e., we shall take $\tilde{\lambda}_{3} \gg \lambda_{233}, \lambda_{333}^{\prime}$, or $\lambda_{233} \gg \tilde{\lambda}_{3}, \lambda_{333}^{\prime}$, or $\lambda_{333}^{\prime} \gg \tilde{\lambda}_{3}, \lambda_{233}$. In order to study the infrared fixed points, we define the following ratios of the dimensionless trilinear couplings and the $S U(3)_{C}$ gauge coupling:

$$
\begin{aligned}
& R_{t}=\frac{h_{t}^{2}}{g_{3}^{2}}, \quad R_{b}=\frac{h_{b}^{2}}{g_{3}^{2}}, \quad R_{\tau}=\frac{h_{\tau}^{2}}{g_{3}^{2}}, \quad R_{\lambda}=\frac{\lambda^{2}}{g_{3}^{2}}, \quad R_{\kappa}=\frac{\kappa^{2}}{g_{3}^{2}}, \\
& \tilde{R}_{3}=\frac{\tilde{\lambda}_{3}^{2}}{g_{3}^{2}}, \quad R=\frac{\lambda_{233}^{2}}{g_{3}^{2}}, \quad R^{\prime}=\frac{\lambda_{333}^{\prime}}{g_{3}^{2}}, \quad R^{\prime \prime}=\frac{\lambda_{233}^{\prime \prime}}{g_{3}^{2}} .
\end{aligned}
$$

We shall first consider the RG evolution of Yukawa couplings in the superpotential (1D), with $B$ violation arising from (3), and $L$ violation arising from the first term in (2). With

the definitions (32) and (33), and retaining only the $S U(3)_{C}$ gauge coupling, the one-loop renormalization group equations for $h_{t}, h_{b}, h_{\tau}, \lambda, k$, and $\tilde{\lambda}_{3}, \lambda_{233}^{\prime \prime}$ can be written in the form

$$
\begin{aligned}
& \frac{d R_{t}}{d\left(-\ln \mu^{2}\right)}=\tilde{\alpha}_{3} R_{t}\left[\left(\frac{16}{3}+b_{3}\right)-6 R_{t}-R_{b}-R_{\lambda}-\tilde{R}_{3}-2 R^{\prime \prime}\right], \\
& \frac{d R_{b}}{d\left(-\ln \mu^{2}\right)}=\tilde{\alpha}_{3} R_{b}\left[\left(\frac{16}{3}+b_{3}\right)-R_{t}-6 R_{b}-R_{\tau}-R_{\lambda}-2 R^{\prime \prime}\right], \\
& \frac{d R_{\tau}}{d\left(-\ln \mu^{2}\right)}=\tilde{\alpha}_{3} R_{\tau}\left[b_{3}-3 R_{b}-4 R_{\tau}-R_{\lambda}-\tilde{R}_{3}\right], \\
& \frac{d R_{\lambda}}{d\left(-\ln \mu^{2}\right)}=\tilde{\alpha}_{3} R_{\lambda}\left[b_{3}-3 R_{t}-3 R_{b}-R_{\tau}-4 R_{\lambda}-2 R_{\kappa}-4 \tilde{R}_{3}\right], \\
& \frac{d R_{\kappa}}{d\left(-\ln \mu^{2}\right)}=\tilde{\alpha}_{3} R_{\kappa}\left[b_{3}-6 R_{\lambda}-6 R_{\kappa}-6 \tilde{R}_{3}\right],
\end{aligned}
$$




$$
\begin{aligned}
& \frac{d \tilde{R}_{3}}{d\left(-\ln \mu^{2}\right)}=\tilde{\alpha}_{3} \tilde{R}_{3}\left[b_{3}-3 R_{t}-R_{\tau}-4 R_{\lambda}-2 R_{\kappa}-4 \tilde{R}_{3}\right] \\
& \frac{d R^{\prime \prime}}{d\left(-\ln \mu^{2}\right)}=\tilde{\alpha}_{3} R^{\prime \prime}\left[\left(8+b_{3}\right)-2 R_{t}-2 R_{b}-6 R^{\prime \prime}\right]
\end{aligned}
$$

where $b_{3}=-3$ is the beta function for $g_{3}$ in NMSSM (or MSSM), and $\tilde{\alpha}_{3}=g_{3}^{2} /\left(16 \pi^{2}\right)$. Choosing the basis of the ratios as $R_{i}=\left(R_{t}, R_{b}, R_{\tau}, R_{\lambda}, R_{\kappa}, R^{\prime \prime}, \tilde{R}_{3}\right)$, we can rewrite the RG equations (34) - (40) in the form $\left(t=-\ln \mu^{2}\right)$

$$
\frac{d R_{i}}{d t}=\tilde{\alpha}_{3} R_{i}\left[\left(r_{i}+b_{3}\right)-\sum_{j} S_{i j} R_{j}\right]
$$

where $r_{i}=\sum_{k} 2 C_{R}, C_{R}$ is the QCD quadratic Casimir for the various fields $\left(C_{Q}=C_{U^{c}}=\right.$ $\left.C_{D^{c}}=4 / 3\right)$ and the sum is over the representation of the three fields associated with the trilinear coupling that enters the definition of $R_{i}$, and $S$ is a matrix whose entries are the numerical coefficients (the wave function anomalous dimensions) of $R_{i}$ 's in the evolution equations (34) - (40). A fixed point is, then, reached when the right hand side of Eq. (41) is 0 for all $i$. If we were to write the fixed point solutions as $R_{i}^{*}$, then there are two fixed point values for each coupling: $R_{i}^{*}=0$, or

$$
\left[\left(r_{i}+b_{3}\right)-\sum_{j} S_{i j} R_{j}^{*}\right]=0 .
$$

It follows that the non-trivial fixed point solution is

$$
R_{i}^{*}=\sum_{j}\left(S^{-1}\right)_{i j}\left(r_{j}+b_{3}\right)
$$

The anomalous dimension matrix $\mathrm{S}$ that enters Eq. (43), which we denote by $S_{B L 1}$ in this case, is easily seen to be

$$
S_{B L 1}=\left[\begin{array}{lllllll}
6 & 1 & 0 & 1 & 0 & 2 & 1 \\
1 & 6 & 1 & 1 & 0 & 2 & 0 \\
0 & 3 & 4 & 1 & 0 & 0 & 1 \\
3 & 3 & 1 & 4 & 2 & 0 & 4 \\
0 & 0 & 0 & 6 & 6 & 0 & 6 \\
2 & 2 & 0 & 0 & 0 & 6 & 0 \\
3 & 0 & 1 & 4 & 2 & 0 & 4
\end{array}\right]
$$

Inverting the matrix (44) and substituting in Eq.(43), we get the following fixed point solution:

$$
\begin{aligned}
R_{t}^{*} & =\frac{29}{76}, \quad R_{b}^{*}=0, \quad R_{\tau}^{*}=-\frac{31}{76}, \quad R_{\lambda}^{*}=\frac{18}{19}, \quad R_{\kappa}^{*}=\frac{33}{38} \\
R^{\prime \prime} * & =\frac{161}{228}, \quad \tilde{R}_{3}^{*}=-\frac{44}{19}
\end{aligned}
$$


We note that $R_{\tau}^{*}, \tilde{R}_{3}^{*}<0$, and, therefore, this fixed point solution is physically unacceptable. We conclude that a simultaneous fixed point for the $B$ and $L$ violating couplings $\lambda_{233}^{\prime \prime}$ and $\tilde{\lambda}_{3}$, and the Yukawa couplings $h_{\tau}, h_{b}, h_{t}, \lambda, \kappa$, does not exist.

Next we consider the RG evolution of Yukawa couplings in the superpotential (1), with $B$ violation arising from (3), and $L$ violation arising from the second term in (2). The one-loop renormalization group equations for $h_{t}, h_{b}, h_{\tau}, \lambda, \kappa$, and $\lambda_{233}$ and $\lambda_{233}^{\prime \prime}$ can be written in the form (41), with the anomalous dimension matrix $S$ given by

$$
S_{B L 2}=\left[\begin{array}{lllllll}
6 & 1 & 0 & 1 & 0 & 0 & 2 \\
1 & 6 & 1 & 1 & 0 & 0 & 2 \\
0 & 3 & 4 & 1 & 0 & 4 & 0 \\
3 & 3 & 1 & 4 & 2 & 0 & 0 \\
0 & 0 & 0 & 6 & 6 & 0 & 0 \\
0 & 0 & 4 & 0 & 0 & 4 & 0 \\
2 & 2 & 0 & 0 & 0 & 0 & 6
\end{array}\right]
$$

where the ordering of the ratios is $R_{i}=\left(R_{t}, R_{b}, R_{\tau}, R_{\lambda}, R_{\kappa}, R, R^{\prime \prime}\right)$. From (46) and (43) we get the fixed point values

$$
\begin{aligned}
& R_{t}^{*}=\frac{1}{2}, \quad R_{b}^{*}=\frac{3}{4}, \quad R_{\tau}^{*}=-\frac{5}{4}, \quad R_{\lambda}^{*}=-\frac{9}{4}, R_{\kappa}^{*}=\frac{7}{4} \\
& R^{*}=\frac{1}{2}, \quad R^{\prime \prime *}=\frac{5}{12} .
\end{aligned}
$$

Since $R_{\tau}^{*}, R_{\lambda}^{*}<0$, this fixed point must also be rejected as being unphysical. Thus, a simultaneous fixed point for the $B$ and $L$ violating couplings $\lambda_{233}^{\prime \prime}$ and $\lambda_{233}$, and the Yukawa couplings $h_{\tau}, h_{b}, h_{t}, \lambda, \kappa$, does not exist.

Finally, we consider the RG evolution of Yukawa couplings in the superpotential (1), with $B$ violation arising from (3), and $L$ violation arising from the third term in (2). The one-loop renormalization group equations for $h_{t}, h_{b}, h_{\tau}, \lambda, \kappa$, and $\lambda_{333}^{\prime}$ and $\lambda_{233}^{\prime \prime}$ can again be written in the form (41), with the anomalous dimension matrix $S$ given by

$$
S_{B L 3}=\left[\begin{array}{lllllll}
6 & 1 & 0 & 1 & 0 & 1 & 2 \\
1 & 6 & 1 & 1 & 0 & 6 & 2 \\
0 & 3 & 4 & 1 & 0 & 3 & 0 \\
3 & 3 & 1 & 4 & 2 & 0 & 0 \\
0 & 0 & 0 & 6 & 6 & 0 & 0 \\
1 & 6 & 1 & 0 & 0 & 6 & 2 \\
2 & 2 & 0 & 0 & 0 & 2 & 6
\end{array}\right]
$$

where the ordering of the ratios is $R_{i}=\left(R_{t}, R_{b}, R_{\tau}, R_{\lambda}, R_{\kappa}, R^{\prime}, R^{\prime \prime}\right)$. This results in the fixed point values

$$
\begin{aligned}
R_{t}^{*} & =\frac{31}{292}, \quad R_{b}^{*}=-\frac{98}{219}, \quad R_{\tau}^{*}=-\frac{285}{292}, \quad R_{\lambda}^{*}=0, \quad R_{\kappa}^{*}=-\frac{1}{2} \\
R^{\prime} * & =\frac{164}{219}, \quad R^{\prime \prime *}=\frac{611}{876}
\end{aligned}
$$


which must also be rejected as being unphysical. We conclude that $B$ and $L$ violating couplings of the highest generation cannot simultaneously approach a non-trivial fixed point in the NMSSM. This is one of the most important conclusions that we draw from the analysis of the renormalization group equations of NMSSM, and is analogous to the corresponding result in MSSM [11].

Given this result, it is now natural to consider the possibility of having either $B$ or $L$ violation, but not both simultaneously, involving the trilinear couplings with highest generation indices in the RG evolution in the NMSSM.

\section{Fixed points with baryon number violation}

In this section we shall consider the infrared fixed points of the NMSSM with $B$ violation. Thus, we shall consider the Yukawa couplings $h_{t}, h_{b}, h_{\tau}, \lambda, \kappa$, and the baryon number violating coupling $\lambda_{233}^{\prime \prime}$. We order the ratios of the trilinear couplings to the gauge coupling $g_{3}$ as $R_{i}=\left(R_{t}, R_{b}, R_{\tau}, R_{\lambda}, R_{\kappa}, R^{\prime \prime}\right)$, resulting in the anomalous dimension matrix

$$
S_{B 1}=\left[\begin{array}{llllll}
6 & 1 & 0 & 1 & 0 & 2 \\
1 & 6 & 1 & 1 & 0 & 2 \\
0 & 3 & 4 & 1 & 0 & 0 \\
3 & 3 & 1 & 4 & 2 & 0 \\
0 & 0 & 0 & 6 & 6 & 0 \\
2 & 2 & 0 & 0 & 0 & 6
\end{array}\right]
$$

leading to the fixed point values

$$
R_{t}^{*}=\frac{9}{16}, \quad R_{b}^{*}=\frac{11}{16}, \quad R_{\tau}^{*}=-\frac{5}{8}, \quad R_{\lambda}^{*}=-\frac{41}{16}, \quad R_{\kappa}^{*}=\frac{33}{16}, \quad R^{\prime \prime *}=\frac{5}{12},
$$

which must be rejected as being unphysical. We are, therefore, constrained to consider fixed point with one of the couplings approaching a zero fixed point value, with all others attaining a non-trivial fixed point value. We first consider a fixed point with $R_{\tau}^{*}=0$, with all others obtaining a non-zero fixed point value. In this case we obtain the fixed point values

$$
R_{\tau}^{*}=0, \quad R_{t}^{*}=\frac{5}{8}, \quad R_{b}^{*}=\frac{5}{8}, \quad R_{\lambda}^{*}=-\frac{23}{8}, R_{\kappa}^{*}=\frac{19}{8}, \quad R^{\prime \prime *}=\frac{5}{12},
$$

which is a physically unacceptable fixed point solution. Proceeding in this manner, it can be shown that there is no acceptable infrared fixed point solution with one of the couplings $h_{t}, h_{b}, h_{\tau}, \lambda, \kappa, \lambda_{233}^{\prime \prime}$ approaching a zero fixed point value, and the remaining approaching a non-zero fixed point value.

We are, therefore, led to the consideration of a fixed point in which two of the Yukawa couplings approach a zero fixed point value, with all others attaining a non-zero fixed point value. We try the fixed point with $R_{\lambda}^{*}=R_{\kappa}^{*}=0$, with the rest approaching a non-zero fixed point value. In this case we obtain the fixed point values

$$
R_{t}^{*}=\frac{31}{292}, \quad R_{b}^{*}=\frac{22}{73}, \quad R_{\tau}^{*}=-\frac{285}{292}, \quad R^{\prime \prime *}=\frac{611}{876},
$$


which must be rejected as a fixed point solution. Taking other pairs of Yukawa couplings to have a zero fixed point values, with the remaining obtaining non-zero values, we find that there is no acceptable fixed point solution in this case.

We next consider the situation in which three of the couplings attain zero fixed point values, with the remaining attaining a non-zero fixed point value. Taking $R_{\tau}^{*}=R_{\lambda}^{*}=R_{\kappa}^{*}=0$, we obtain the fixed point

$$
R_{t}^{*}=\frac{2}{17}, \quad R_{b}^{*}=\frac{2}{17}, \quad R^{\prime \prime *}=\frac{77}{102},
$$

which is a physically acceptable fixed point solution. We find that there is no other acceptable fixed point solution with three of the couplings obtaining fixed point values, with the remaining approaching a non-trivial fixed point value.

We now consider the case when four of the trilinear Yukawa couplings approach a trivial fixed point, with the remaining two having a non-trivial fixed point value. In this case we find the following acceptable fixed point solutions:

$$
R_{\tau}^{*}=R_{\lambda}^{*}=R_{\kappa}^{*}=R^{\prime \prime *}=0, \quad R_{t}^{*}=\frac{1}{3}, R_{b}^{*}=\frac{1}{3}
$$

and

$$
R_{\tau}^{*}=R_{\lambda}^{*}=R_{\kappa}^{*}=R_{b}^{*}=0, R_{t}^{*}=\frac{1}{8}, R^{\prime \prime *}=\frac{19}{24}
$$

Since there are more than one theoretically acceptable IRFPs in this case, it is necessary to determine, which, if any, of these fixed points is more likely to be realized in nature. To this end, we must examine the stability of each of the fixed point solutions (54), (55) and (56).

The infra-red stability of a fixed point is determined by the sign of the quantitites

$$
\lambda_{i}=\frac{1}{b_{3}}\left[\sum_{j=m+1}^{n} S_{i j} R_{j}^{*}-\left(r_{i}+b_{3}\right)\right],
$$

for those couplings which have a fixed point value zero, $R_{i}^{*}=0, i=1,2, \ldots, m$, and by the sign of the eigenvalues of the matrix

$$
A_{i j}=\frac{1}{b_{3}} R_{i}^{*} S_{i j}, i=m+1, \ldots, n,
$$

for those couplings which have a non-trivial IRFP [5], where $R_{i}^{*}$ is the set of the nontrivial fixed point values of the Yukawa couplings under consideration, and $S_{i j}$ is the matrix appearing in the corresponding renormalization group equations (41) for the ratios $R_{i}$. For stability, we require all the $\lambda_{i}$ 's to have negative sign, and the eigenvalues of the matrix (58) to have negative real parts (note that the QCD $\beta$-function $b_{3}$ is negative). For the infra-red fixed point (54), we find from Eq. (57)

$$
\lambda_{1}=-\frac{19}{17}, \quad \lambda_{2}=-\frac{21}{17}, \quad \lambda_{3}=-1
$$


corresponding to $R_{\tau}^{*}=0, R_{\lambda}^{*}=0$, and $R_{\kappa}^{*}=0$, respectively and for the eigenvalues of the matrix (58)

$$
\lambda_{4}=-\frac{10}{51} \simeq-0.2, \quad \lambda_{5}=\frac{-273-\sqrt{43113}}{306} \simeq-1.6, \quad \lambda_{6}=\frac{-273+\sqrt{43113}}{306} \simeq-0.2
$$

corresponding to the non-trivial fixed point values for $R^{\prime \prime *}, R_{b}^{*}$ and $R_{t}^{*}$, respectively. Since all the $\lambda_{i}, i=1,2,3,4,5,6$ are negative, the fixed point (54) is infra-red stable.

Next we consider the stability of the fixed point (55). Since in this case $R_{\lambda}^{*}=R_{\kappa}^{*}=$ $R_{\tau}^{*}=R^{\prime \prime *}=0$, we have to obtain the behaviour of these couplings around the origin. This behaviour is determined by the quantities (57) which, in this case, are

$$
\lambda_{1}=-\frac{4}{3}, \lambda_{2}=-\frac{5}{3}, \lambda_{3}=-1 \lambda_{4}=\frac{11}{9}
$$

thereby indicating that the fixed point is unstable in the infra-red region. For completeness we also obtain the behaviour of $R_{b}$ and $R_{t}$ around their respective fixed points governed by the corresponding eigenvalues of the matrix (58). We obtain for the eigenvalues of this matrix

$$
\lambda_{5}=-\frac{5}{9}, \lambda_{6}=-\frac{7}{9}
$$

Although $\lambda_{5}, \lambda_{6}$ are negative, the fact that $\lambda_{4}>0$ implies that the fixed point (55) is unstable in the infra-red region. Thus, this infra-red fixed point with trivial fixed point value for the baryon number violating coupling $\lambda_{233}$ will never be realized at low energies, and must be rejected. Similarly, it is straightforward to see that the fixed point (56) is unstable in the infra-red, and must be rejected.

One may also consider the case where the couplings $\lambda_{233}^{\prime \prime *}, \kappa, \lambda, h_{\tau}, h_{b}$ attain trivial fixed point values, whereas $h_{t}$ attains a non-trivial fixed point value. In this case we find $R_{t}^{*}=$ 7/18, the well-known Pendleton-Ross type fixed point [3]. The stability of this fixed point solution is obtained by simply considering the quantities (57)

$$
\lambda_{i}=\frac{1}{b_{3}}\left[\left(S_{B 1}\right)_{i 6} R_{6}^{*}-\left(r_{i}+b_{3}\right)\right], \quad i=1,2,3,4,5,
$$

where the matrix $S_{B 1}$ is the one which appears in (50) but with the ordering of the ratios as $R_{i}=\left(R_{\tau}, R_{\lambda}, R_{\kappa}, R_{b}, R^{\prime \prime}, R_{t}\right)$, which yields

$$
\lambda_{1}=-1, \lambda_{2}=-\frac{25}{18}, \lambda_{3}=-1, \lambda_{3}=\frac{35}{54}, \lambda_{5}=\frac{38}{27}
$$

thereby rendering this fixed point unstable.

Finally, one may consider the case where $R^{\prime \prime *}=0$, with $R_{t}, R_{b}, R_{\tau}, R_{\lambda}, R_{\kappa}$, attaining non-trivial fixed point values. This is the case of the NMSSM with $R_{p}$ conservation and with all the third generation Yukawa couplings taken into account. In this case, we find the fixed point solution:

$$
R_{t}^{*}=\frac{37}{48}, R_{b}^{*}=\frac{33}{48} R_{\tau}^{*}=-\frac{5}{8}, R_{\lambda}^{*}=-\frac{51}{16}, R_{k}^{*}=\frac{43}{16},
$$


which must be rejected as being unphysical.

Thus, we have shown that the only fixed point which is stable in the infra red region is the baryon number, and $R_{p}$, violating solution (54). We note that the value of $R_{t}^{*}$ corresponding to this solution is lower than the fixed point value of $7 / 18$ for NMSSM (and MSSM) with baryon number, and $R_{p}$, conservation.

\section{Fixed points with lepton number violation}

In order to investigate the possibility of having a stable fixed point with lepton number violation, we now study the renormalization group evolution for the lepton number, and $R_{p}$, violating couplings in the superpotential (2). We shall consider the trilinear couplings $\tilde{\lambda}_{3}, \lambda_{233}$ and $\lambda_{333}^{\prime}$, together with the Yukawa couplings $h_{t}, h_{b}, h_{\tau}, \lambda, \kappa$. As indicated in Sec. II, we shall assume, without loss of generality, that there is a hierarchy of lepton number violating couplings, and consider only one lepton number violating coupling at a time. Thus, we shall consider three different cases, i.e., we shall take either $\tilde{\lambda}_{3} \gg \lambda_{233}, \lambda_{333}^{\prime}$, or $\lambda_{233} \gg \tilde{\lambda}_{3}, \lambda_{333}^{\prime}$, or $\lambda_{333}^{\prime} \gg \tilde{\lambda}_{3}, \lambda_{233}$, respectively.

We first consider the case when $\tilde{\lambda}_{3}$ is the dominant lepton number violating coupling. Ordering the ratios of the squares of the trilinear couplings to the square of the gauge coupling $g_{3}$ as $R_{i}=\left(R_{t}, R_{b}, R_{\tau}, R_{\lambda}, R_{\kappa}, \tilde{R}_{3}\right)$, the anomalous dimension matrix, $S_{L 11}$, in this case is given by

$$
S_{L 11}=\left[\begin{array}{llllll}
6 & 1 & 0 & 1 & 0 & 1 \\
1 & 6 & 1 & 1 & 0 & 0 \\
0 & 3 & 4 & 1 & 0 & 1 \\
3 & 3 & 1 & 4 & 2 & 4 \\
0 & 0 & 0 & 6 & 6 & 6 \\
3 & 0 & 1 & 4 & 2 & 4
\end{array}\right]
$$

leading to the fixed point values

$$
R_{t}^{*}=\frac{32}{45}, R_{b}^{*}=0, R_{\tau}^{*}=-\frac{4}{15}, R_{\lambda}^{*}=\frac{17}{9}, R_{\kappa}^{*}=\frac{43}{30}, \tilde{R}_{3}^{*}=-\frac{172}{45} .
$$

Since $R_{\tau}^{*}, \tilde{R}_{3}^{*}<0$, this fixed point is unacceptable. Thus, there is no nontrivial infrared fixed point for both the lepton number violating coupling $\tilde{\lambda}_{3}$, and all other trilinear couplings in the NMSSM.

Next we consider we consider a trivial fixed point for one of the couplings, and nontrivial one for the. Taking $R_{b}^{*}=0$, which is relevant for low values of $\tan \beta$, the anomalous dimension matrix is

$$
S_{L 12}=\left[\begin{array}{lllll}
6 & 0 & 1 & 0 & 1 \\
0 & 4 & 1 & 0 & 1 \\
3 & 1 & 4 & 2 & 4 \\
0 & 0 & 6 & 6 & 6 \\
3 & 1 & 4 & 2 & 4
\end{array}\right]
$$


which is singular. This solution corresponds to a fixed line or a surface 1 . Continuing in this manner, and taking each one of the couplings to have a trivial fixed point value with the rest a nontrivial fixed point value, we find there is no acceptable infrared fixed point in this case.

We now try to obtain a fixed point with two of the couplings approaching a trivial fixed point, while the rest having a non-trivial fixed point. Taking $R_{\lambda}^{*}=R_{\kappa}^{*}=0$, we obtain the fixed point values for the remaining couplings

$$
R_{t}^{*}=\frac{11}{24}, \quad R_{b}^{*}=\frac{11}{24}, R^{*} \tau=-\frac{7}{8}, \tilde{R}_{3}^{*}=-\frac{7}{8},
$$

which is an unacceptable fixed point. Continuing in this manner we find that there are no acceptable infrared fixed points in this case. We have also checked that for the case with three or four of the couplings approaching a zero fixed point value, with the remaining having nontrivial fixed point values, there are no physically acceptable fixed point solutions. Finally, for the case with all the couplings, except the top-quark Yukawa coupling, approaching a trivial fixed point value, we have

$$
R_{b}^{*}=R_{\tau}^{*}=R_{\lambda}^{*}=R_{\kappa}^{*}=\tilde{R}_{3}^{*}=0, R_{t}^{*}=\frac{7}{18}
$$

which is an acceptable fixed point. However, it is easily seen that this fixed point is unstable in the infrared region. We, therefore, conclude that for the case with lepton number violation through the coupling $\tilde{\lambda}_{3}$, there are no acceptable infra-red fixed points.

If on the other hand the coupling $\lambda_{233}$ is the dominant of the lepton number couplings, then the anomalous dimension matrix is given by

$$
S_{L_{21}}=\left[\begin{array}{llllll}
6 & 1 & 0 & 1 & 0 & 0 \\
1 & 6 & 1 & 1 & 0 & 0 \\
0 & 3 & 4 & 1 & 0 & 4 \\
3 & 3 & 1 & 4 & 2 & 0 \\
0 & 0 & 0 & 6 & 6 & 0 \\
0 & 0 & 4 & 0 & 0 & 4
\end{array}\right]
$$

in the basis $R_{i}=\left(R_{t}, R_{b}, R_{\tau}, R_{\lambda}, R_{\kappa}, R\right)$, leading to the fixed point values

$$
R_{t}^{*}=\frac{17}{24}, R_{b}^{*}=\frac{23}{24}, R_{\tau}^{*}=-\frac{5}{4}, R_{\lambda}=-\frac{23}{8}, R_{\kappa}^{*}=\frac{19}{8} R^{*}=\frac{1}{2},
$$

which must be rejected as a fixed point. We can try with one of the couplings, namely $R_{b}^{*}$ approaching a trivial fixed point value with the rest approaching a nontrivial fixed point. This case is relevant for low values of $\tan \beta$. We get

$$
R_{b}^{*}=0, R_{t}^{*}=\frac{7}{18}, R_{\tau}^{*}=-\frac{19}{6}, R_{\lambda}=0, R_{\kappa}^{*}=-\frac{1}{2} R^{*}=\frac{29}{12},
$$

\footnotetext{
${ }^{1}$ Here we correct an error in [13]
} 
which is also unacceptable. We may also try a fixed point with $R_{\tau}^{*}=0$, and a non-trivial fixed point for other couplings. In this case we get

$$
R_{\tau}^{*}=0, R_{t}^{*}=\frac{5}{6}, R_{b}^{*}=\frac{5}{6}, R_{\lambda}=-\frac{7}{2}, R_{\kappa}^{*}=3 R^{*}=-\frac{3}{4},
$$

making this also an unacceptable fixed point. Continuing in this fashion, we find that there are no fixed points with one of the couplings approaching a zero fixed point value, with the rest having non-trivial fixed point values.

We now try to obtain fixed points with two of the couplings having a fixed point value zero, and the rest having non-zero fixed point values. Taking $R_{b}^{*}=R_{\tau}^{*}=0$, we obtain

$$
R_{t}^{*}=\frac{20}{27}, R_{\lambda}=-\frac{19}{9}, R_{\kappa}^{*}=\frac{29}{18} R^{*}=-\frac{3}{4},
$$

which is unphysical, and hence unacceptable. Similarly, we have checked there is no physically acceptable fixed point with any of the two couplings approaching a trivial fixed point value, with the rest attaining a non-trivial fixed point value. Continuing in this fashion, we find that there are no acceptable fixed points in the infrared region for the lepton number violating coupling $\lambda_{233}$. In particular, the fixed point with five of the couplings approaching a trivial fixed point value

$$
R_{b}^{*}=R_{\tau}^{*}=R_{\lambda}=R_{\kappa}^{*}=R^{*}=0,
$$

and $R_{t}$ approaching a non-trivial fixed point value, $R_{t}^{*}=7 / 18$, is unstable in the infrared region.

Finally, we consider the case when the coupling $\lambda_{333}^{\prime}$ is the dominant of the lepton number violating couplings. With the ordering of the couplings $R_{i}=\left(R_{t}, R_{b}, R_{\tau}, R_{\lambda}, R_{\kappa}, R^{\prime}\right)$, the anomalous dimension matrix in this case is

$$
S_{L 31}=\left[\begin{array}{llllll}
6 & 1 & 0 & 1 & 0 & 1 \\
1 & 6 & 1 & 1 & 0 & 6 \\
0 & 3 & 4 & 1 & 0 & 3 \\
3 & 3 & 1 & 4 & 2 & 0 \\
0 & 0 & 0 & 6 & 6 & 0 \\
1 & 6 & 1 & 0 & 0 & 0
\end{array}\right],
$$

leading to the fixed point values

$$
R_{t}^{*}=\frac{55}{183}, R_{b}^{*}=-\frac{107}{183}, R_{\tau}^{*}=-\frac{70}{61}, R_{\lambda}=0, R_{\kappa}^{*}=-\frac{1}{2}, R^{* *}=\frac{68}{61},
$$

which are unacceptable. As in the other cases, we try a fixed point with one of the couplings approaching a zero fixed point value, and the rest non-trivial values. Taking, $R_{b}^{*}=0$, we have

$$
R_{t}^{*}=\frac{121}{210}, R_{\tau}^{*}=-\frac{47}{70}, R_{\lambda}=-\frac{107}{70}, R_{\kappa}^{*}=\frac{36}{35}, R^{*}=\frac{17}{42},
$$

which is an unacceptable fixed point. Continuing in this manner, we find that in this case there are only two acceptable infrared fixed points: 


$$
R_{b}^{*}=R_{\tau}^{*}=R_{\lambda}=R_{\kappa}^{*}=0, \quad R_{t}^{*}=\frac{1}{3}, R^{* *}=\frac{1}{3}
$$

and

$$
R_{\tau}^{*}=R_{\lambda}=R_{\kappa}^{*}=R^{\prime}=0, \quad R_{t}^{*}=\frac{1}{3}, R_{b}^{*}=\frac{1}{3} .
$$

In order to determine which of these fixed points, if any, is actually realised, we need to check the stability of these fixed points. We first consider the fixed point (80). In this case the quantities (57) are calculated to be

$$
\lambda_{1}=0, \lambda_{2}=-\frac{4}{3}, \lambda_{3}=-\frac{4}{3}, \lambda_{4}=-1
$$

corresponding to the the zero fixed point values in (80). From (82) we conclude that the fixed point $(80)$ will never be reached in the infrared region. The fixed point is either a saddle point or an ultra-violet istable fixed point.

The stability of the fixed point (81) is determined in a manner analogous to that of the fixed point (80). We find that the fixed point (81) is either a saddle point, or an ultra-violet stable fixed point. That the stability properties of the fixed points (80) and (81) are identical is a consequence of the symmetry of the renormalization group equations (14) - (22). We conclude that there are no non-trivial stable fixed points in the infra-red region for the $L$ violating coupling $\lambda_{333}^{\prime}$.

To sum up, we have found that there are no IRSFPs in the NMSSM with the highest generation lepton number violation. This result, together with the result on the fixed point with baryon number violation, shows that only the simultaneous non-trivial fixed point (54) for the baryon number violating coupling $\lambda_{233}^{\prime \prime}$, and the top- and bottom-quark Yukawa couplings, $h_{t}$ and $h_{b}$, is stable in the infra-red region. This result is analogous to the result obtained in MSSM, and shows that the additional couplings in NMSSM have no effect on the infrared fixed point behavior of the Yukawa and baryon number violating couplings.

It is appropriate to examine the implications of the value of the top-quark mass predicted by our fixed point analysis. From (54), it is readily seen that the fixed point value for the top-quark Yukawa coupling translates into a top-quark (pole) mass of about $m_{t} \simeq 70 \sin \beta$ $\mathrm{GeV}$, which is incompatible with the measured value of [19] of top mass, $m_{t} \simeq 174 \mathrm{GeV}$, for any value of $\tan \beta$. It follows that the true fixed point obtained here provides only a lower bound on the baryon number violating coupling $\lambda_{233}^{\prime \prime} \gtrsim 0.97$.

\section{Infrared fixed points for the trilinear soft supersymmetry breaking parameters}

Having obtained the infrared fixed point structure of the Yukawa couplings of NMSSM with baryon and lepton number violation, we now consider the renormalization group evolution and the fixed point structure for the soft supersymmetry breaking trilinear parameters $A_{i}$. Since there is only one IRSFP in this case, we shall consider the IRFPs for the $A$ parameters corresponding to this case only, i.e. for $A_{t}, A_{b}, A_{\tau}, A_{\lambda}, A_{\kappa}$, and $A_{\lambda_{233}^{\prime \prime}}$.

Retaining only these parameters, and with the definitions $\tilde{A}_{i}=A_{i} / M_{3}\left(A_{i}=\right.$ $\left.A_{t}, A_{b}, A_{\tau}, A_{\lambda}, A_{\kappa}, A_{\lambda_{23}^{\prime \prime}}\right)$, we obtain from Eq. (23) - Eq. (31) the renormalization group equations for the relevant $\tilde{A}_{i}$ (neglecting the $S U(2)_{L}$ and $U(1)_{Y}$ gauge couplings): 


$$
\begin{aligned}
& \frac{d \tilde{A}_{t}}{d\left(-\ln \mu^{2}\right)}=\tilde{\alpha}_{3}\left[\frac{16}{3}-\left(6 R_{t}-b_{3}\right) \tilde{A}_{t}-R_{b} \tilde{A}_{b}-R^{\prime \prime} \tilde{A}_{\lambda_{233}^{\prime \prime}}\right], \\
& \frac{d \tilde{A}_{b}}{d\left(-\ln \mu^{2}\right)}=\tilde{\alpha}_{3}\left[\frac{16}{3}-R_{t} \tilde{A}_{t}-\left(6 R_{b}-b_{3}\right) \tilde{A}_{b}-R_{\tau} \tilde{A}_{\tau}-R_{\lambda} \tilde{A}_{\lambda}-2 R^{\prime \prime} \tilde{A}_{\lambda_{233}^{\prime \prime}}\right], \\
& \frac{d \tilde{A}_{\tau}}{d\left(-\ln \mu^{2}\right)}=\tilde{\alpha}_{3}\left[-3 R_{b} \tilde{A}_{b}-\left(4 R_{\tau}-b_{3}\right) \tilde{A}_{\tau}-R_{\lambda} \tilde{A}_{\lambda}\right], \\
& \frac{d \tilde{A}_{\lambda}}{d\left(-\ln \mu^{2}\right)}=\tilde{\alpha}_{3}\left[-3 R_{t} \tilde{A}_{t}-3 R_{b} \tilde{A}_{b}-R_{\tau} \tilde{A}_{\tau}-\left(4 R_{\lambda}-b_{3}\right) \tilde{A}_{\lambda}-2 R_{\kappa} \tilde{A}_{\kappa}\right], \\
& \frac{d \tilde{A}_{\kappa}}{d\left(-\ln \mu^{2}\right)}=\tilde{\alpha}_{3}\left[-6 R_{\lambda} \tilde{A}_{\lambda}-\left(6 R_{\kappa}-b_{3}\right) \tilde{A}_{\kappa}\right], \\
& \frac{d \tilde{A}_{\lambda_{23}^{\prime \prime}}}{d\left(-\ln \mu^{2}\right)}=\tilde{\alpha}_{3}\left[8-2 R_{t} \tilde{A}_{t}-2 R_{b} \tilde{A}_{b}-\left(6 R^{\prime \prime}-b_{3}\right) \tilde{A}_{\lambda_{233}^{\prime \prime}}\right],
\end{aligned}
$$

which can be written in a compact form

$$
\frac{d \tilde{A}_{i}}{d\left(-\ln \mu^{2}\right)}=\tilde{\alpha}_{3}\left[r_{i}-\sum_{j} K_{i j} \tilde{A}_{j}\right],
$$

in the basis $\tilde{A}_{i}=\left(\tilde{A}_{\tau}, \tilde{A}_{\lambda}, \tilde{A}_{\kappa}, \tilde{A}_{\lambda_{233}^{\prime \prime}}, \tilde{A}_{b}, \tilde{A}_{t}\right)$, where $r_{i}=(0,0,0,8,16 / 3,16 / 3)$, and where $K$ is a matrix whose entries are fully specified by the wave function anomalous dimensions and $R_{i}$. A fixed point for $\tilde{A}_{i}$ is, then, reached when the right hand side of Eq. (89) vanishes for all $i$. Denoting this fixed point solution by $\tilde{A}_{i}^{*}$, we have

$$
r_{i}-\sum_{j} K_{i j}^{*} \tilde{A}_{j}^{*}=0,
$$

where $K^{*}$ is the matrix $K$ evaluated when $R_{i}$ take their fixed point values $R_{i}^{*}$. From Eq. (83) - Eq. (88) we see that the matrix $K$ at the fixed point can be written as

$$
K^{*}=\left[\begin{array}{cccccc}
4 R_{\tau}^{*}-b_{3} & R_{\lambda}^{*} & 0 & 0 & 3 R_{b}^{*} & 0 \\
R_{\tau}^{*} & \left(4 R_{\lambda}^{*}-b_{3}\right) & 2 R_{\kappa}^{*} & 0 & 3 R_{b}^{*} & 3 R_{t}^{*} \\
0 & 6 R_{\lambda}^{*} & \left(6 R_{\kappa}^{*}-b_{3}\right) & 0 & 0 & 0 \\
0 & 0 & 0 & \left(6 R^{\prime \prime *}-b_{3}\right) & 2 R_{b}^{*} & 2 R_{t}^{*} \\
R_{\tau}^{*} & R_{\lambda}^{*} & 0 & 2 R^{\prime \prime *} & \left(6 R_{b}^{*}-b_{3}\right) & R_{t}^{*} \\
0 & 0 & 0 & 2 R^{\prime \prime *} & R_{b}^{*} & \left(6 R_{t}^{*}-b_{3}\right)
\end{array}\right]
$$

with $R_{\tau}^{*}=R_{\lambda}^{*}=R_{\kappa}^{*}=0$, and $R^{\prime \prime *}, R_{b}^{*}, R_{t}^{*}$ given by their fixed point values in (54). The fixed points $\tilde{A}_{i}^{*}$ are given by the solution of

$$
\tilde{A}_{i}^{*}=\sum_{j}\left(K^{*-1}\right)_{i j} r_{j}
$$

with the result

$$
\tilde{A}_{\tau}^{*}=-\frac{2}{17}, \tilde{A}_{\lambda}^{*}=-\frac{4}{17}, \tilde{A}_{\kappa}^{*}=0, \tilde{A}_{\lambda_{333}^{\prime \prime}}^{*}=\tilde{A}_{b}^{*}=\tilde{A}_{t}^{*}=1
$$


We have carried out the stability analysis of the fixed point (93) in a manner analogous to that of the Yukawa couplings in the previous subsection, and find it to be infra-red stable.

We note that the fixed point values of $\tilde{A}_{\tau}, \tilde{A}_{\lambda_{23}^{\prime \prime}}, \tilde{A}_{b}, \tilde{A}_{t}$ in (93) are the same as in MSSM with baryon number violation [11].

\section{QUASI-FIXED POINTS}

The infrared fixed points that we have discussed in the previous section are the true IRFPs of the renormalization group equations. However, these fixed points are not reached in practice, the range between the large(GUT) scale and the weak scale being too small for the ratios to closely approach the fixed point values. In that case, the various couplings may be determined by the quasi-fixed point behaviour [4, where the value of various couplings at the weak scale is independent of its value at the large(GUT) scale, provided the couplings at the unification scale are large. In this section, we shall discuss the quasi-fixed point behaviour of the Yukawa couplings and the $A$ parameters of the NMSSM with $B$ violation, corresponding to the true fixed points that we have obtained in the previous section.

In order to discuss the quasi-fixed points, we shall first write down the analytical solution for the trilinear couplings of the NMSSM in a closed form. Since the simultaneous fixed point for the third generation Yukawa couplings, $\lambda, \kappa$, and the baryon number violating coupling $\lambda_{233}^{\prime \prime}$ is stable, we shall consider the quasi-fixed points for these couplings only. For this purpose we define

$$
\begin{gathered}
\tilde{Y}_{t}=\frac{h_{t}^{2}}{16 \pi^{2}}, \quad \tilde{Y}_{b}=\frac{h_{b}^{2}}{16 \pi^{2}}, \quad \tilde{Y}_{\tau}=\frac{h_{\tau}^{2}}{16 \pi^{2}}, \\
\tilde{Y}_{\lambda}=\frac{\lambda^{2}}{16 \pi^{2}}, \quad \tilde{Y}_{\kappa}=\frac{\kappa^{2}}{16 \pi^{2}}, \\
\tilde{Y}^{\prime \prime}=\frac{\lambda_{233}^{\prime \prime 2}}{16 \pi^{2}} .
\end{gathered}
$$

Then the solution of the RG equations (14) - (18), and (22) for the Yukawa, the trilinear couplings $\lambda$ and $\kappa$, and the $B$ violating couplings can be written in a closed form 20]

$$
\tilde{Y}_{i}(t)=\frac{\tilde{Y}_{i}(0) F_{i}(t)}{1+a_{i i} \tilde{Y}_{i}(0) \int_{0}^{t} F_{i}\left(t^{\prime}\right) d t^{\prime}}, \quad t=\ln \left(\frac{M_{G}^{2}}{\mu^{2}}\right),
$$

where $M_{G}$ is some large initial scale, and where $\tilde{Y}_{i}$ stands for the functions $\tilde{Y}_{t}, \tilde{Y}_{b}, \tilde{Y}_{\tau}, \tilde{Y}_{\lambda}, \tilde{Y}_{\kappa}$, and $\tilde{Y}^{\prime \prime}$, respectively. Analogous notation holds for the functions $F_{i}$. The quantities $a_{i i}$ are the diagonal elements of the wave function anamolous dimension matrix, and are given by

$$
a_{i i}=\{6,6,4,4,6,6\}
$$

and the functions $F_{i}$ are given by the set of integral equations 


$$
\begin{aligned}
F_{t}(t) & =\frac{E_{t}(t)}{\left(1+6 \tilde{Y}_{b}(0) \int_{0}^{t} F_{b}\left(t^{\prime}\right) d t^{\prime}\right)^{1 / 6}\left(1+4 \tilde{Y}_{\lambda}(0) \int_{0}^{t} F_{\lambda}\left(t^{\prime}\right) d t^{\prime}\right)^{1 / 4}\left(1+6 \tilde{Y}^{\prime \prime}(0) \int_{0}^{t} F^{\prime \prime}\left(t^{\prime}\right) d t^{\prime}\right)^{1 / 3}}, \\
F_{b}(t) & =\frac{E_{b}(t)}{\left(1+6 \tilde{Y}_{t}(0) \int_{0}^{t} F_{t}\left(t^{\prime}\right) d t^{\prime}\right)^{1 / 6}\left(1+4 \tilde{Y}_{\tau}(0) \int_{0}^{t} F_{\tau}\left(t^{\prime}\right) d t^{\prime}\right)^{1 / 4}\left(1+4 \tilde{Y}_{\lambda}(0) \int_{0}^{t} F_{\lambda}\left(t^{\prime}\right) d t^{\prime}\right)^{1 / 4}} \\
& \times \frac{1}{\left(1+6 \tilde{Y}^{\prime \prime}(0) \int_{0}^{t} F^{\prime \prime}\left(t^{\prime}\right) d t^{\prime}\right)^{1 / 3}}, \\
F_{\tau}(t) & =\frac{E_{\tau(t)}}{\left(1+6 \tilde{Y}_{b}(0) \int_{0}^{t} F_{b}\left(t^{\prime}\right) d t^{\prime}\right)^{1 / 2}\left(1+4 \tilde{Y}_{\lambda}(0) \int_{0}^{t} F_{\lambda}\left(t^{\prime}\right) d t^{\prime}\right) 1 / 4}, \\
F_{\lambda}(t) & =\frac{E_{\lambda}(t)}{\left(1+6 \tilde{Y}_{t}(0) \int_{0}^{t} F_{t}\left(t^{\prime}\right) d t^{\prime}\right)^{1 / 2}\left(1+6 \tilde{Y}_{b}(0) \int_{0}^{t} F_{b}\left(t^{\prime}\right) d t^{\prime}\right)^{1 / 2}\left(1+4 \tilde{Y}_{\tau}(0) \int_{0}^{t} F_{\tau}\left(t^{\prime}\right) d t^{\prime}\right)^{1 / 4}} \\
& \times \frac{1}{\left(1+6 \tilde{Y}_{\kappa}(0) \int_{0}^{t} F_{\kappa}\left(t^{\prime}\right) d t^{\prime}\right)^{1 / 3}}, \\
F_{\kappa}(t) & =\frac{E_{\kappa}(t)}{\left(1+4 \tilde{Y}_{\lambda}(0) \int_{0}^{t} F_{\lambda}\left(t^{\prime}\right) d t^{\prime}\right)^{3 / 2}}, \\
F^{\prime \prime}(t) & =\frac{E^{\prime \prime}(t)}{\left(1+6 \tilde{Y}_{t}(0) \int_{0}^{t} F_{t}\left(t^{\prime}\right) d t^{\prime}\right)^{1 / 3}\left(1+6 \tilde{Y}_{b}(0) \int_{0}^{t} F_{t}\left(t^{\prime}\right) d t^{\prime}\right)^{1 / 3}},
\end{aligned}
$$

where the functions $E_{i}(t)\left(=E_{t}(t), E_{b}(t), E_{\tau}(t), E_{\lambda}(t), E_{\kappa}(t)\right.$ and $\left.E^{\prime \prime}(t)\right)$ are given by

$$
E_{j}(t)=\prod_{m=1}^{3}\left(1+b_{m} \tilde{\alpha}_{m}(0) t\right)^{c_{j m} / b_{m}}
$$

with

$$
\begin{gathered}
\tilde{\alpha}_{m}(0)=\frac{g_{m}^{2}(0)}{16 \pi^{2}}, m=1,2,3, \\
c_{t m}=\left(\frac{13}{15}, 3, \frac{16}{3}\right), \quad c_{b m}=\left(\frac{7}{15}, 3, \frac{16}{3}\right), \quad c_{\tau m}=\left(\frac{9}{5}, 3,0\right), \\
c_{\lambda i}=\left(\frac{3}{5}, 3,0\right), \quad c_{\kappa i}=(0,0,0), \quad c_{\lambda_{233}^{\prime \prime} i}=\left(\frac{4}{5}, 0,8\right) .
\end{gathered}
$$

The solutions for the RG equations for the gauge couplings (12) and the gaugino masses (13) are well known and will not be repeated here. We note that (97) gives the exact solution for the Yukawa couplings, while $F_{i}$ 's in (99) - (104) should in principle be solved iteratively.

In the regime where the Yukawa couplings $\tilde{Y}_{t}(0), \tilde{Y}_{b}(0), \tilde{Y}_{\tau}(0), \tilde{Y}_{\lambda}(0), \tilde{Y}_{\kappa}(0), \tilde{Y}^{\prime \prime}(0) \rightarrow$ $\infty$ with their ratios fixed, it is legitimate to drop 1 in the denominators of the equations (97) and (99) - (104), so that the exact solutions for the trilinear couplings approach the infrared quasi-fixed-point (IRQFP) defined by

$$
\tilde{Y}_{i}^{Q F P}(t)=\frac{F_{i}^{Q F P}(t)}{a_{i i} \int_{0}^{t} F_{i}^{Q F P}\left(t^{\prime}\right) d t^{\prime}},
$$


with

$$
\begin{aligned}
F_{t}^{Q F P}(t) & =\frac{E_{t}(t)}{\left(\int_{0}^{t} F_{b}^{Q F P}\left(t^{\prime}\right) d t^{\prime}\right)^{1 / 6}\left(\int_{0}^{t} F_{\lambda}^{Q F P}\left(t^{\prime}\right) d t^{\prime}\right)^{1 / 4}\left(\int_{0}^{t} F^{\prime \prime Q F P}\left(t^{\prime}\right) d t^{\prime}\right)^{1 / 3}}, \\
F_{b}^{Q F P}(t) & =\frac{E_{b}(t)}{\left(\int_{0}^{t} F_{t}^{Q F P}\left(t^{\prime}\right) d t^{\prime}\right)^{1 / 6}\left(\int_{0}^{t} F_{\tau}^{Q F P}\left(t^{\prime}\right) d t^{\prime}\right)^{1 / 4}\left(\int_{0}^{t} F_{\lambda}^{Q F P}\left(t^{\prime}\right) d t^{\prime}\right)^{1 / 4}\left(\int_{0}^{t} F^{\prime \prime Q F P}\left(t^{\prime}\right) d t^{\prime}\right)^{1 / 3}}, \\
F_{\tau}^{Q F P}(t) & =\frac{E_{\tau(t)}}{\left(\int_{0}^{t} F_{b}^{Q F P}\left(t^{\prime}\right) d t^{\prime}\right)^{1 / 2}\left(\int_{0}^{t} F_{\lambda}^{Q F P}\left(t^{\prime}\right) d t^{\prime}\right) 1 / 4}, \\
F_{\lambda}^{Q F P}(t) & =\frac{E_{\lambda}(t)}{\left(\int_{0}^{t} F_{t}^{Q F P}\left(t^{\prime}\right) d t^{\prime}\right)^{1 / 2}\left(\int_{0}^{t} F_{b}\left(t^{\prime}\right)^{Q F P} d t^{\prime}\right)^{1 / 2}\left(\int_{0}^{t} F_{\tau}\left(t^{\prime}\right)^{Q F P} d t^{\prime}\right)^{1 / 4}\left(\int_{0}^{t} F_{\kappa}^{Q F P}\left(t^{\prime}\right) d t^{\prime}\right)^{1 / 3}}, \\
F_{\kappa}^{Q F P}(t) & =\frac{E_{\kappa}(t)}{\left(\int_{0}^{t} F_{\lambda}^{Q F P}\left(t^{\prime}\right) d t^{\prime}\right)^{3 / 2}}, \\
F^{\prime \prime Q F P}(t) & =\frac{E^{\prime \prime}(t)}{\left(\int_{0}^{t} F_{t}^{Q F P}\left(t^{\prime}\right) d t^{\prime}\right)^{1 / 3}\left(\int_{0}^{t} F_{b}^{Q F P}\left(t^{\prime}\right) d t^{\prime}\right)^{1 / 3}} .
\end{aligned}
$$

We stress here that both the dependence on the initial conditions for each Yukawa coupling as well as the dependence on the ratios of initial values of Yukawa couplings have completely dropped out of the runnings in Eqs. (109) and (110) - (115). In other words, the quasi-fixedpoints (109) are independent of whether the $B$ and $L$ violating couplings and the third generation Yukawa couplings are unified or not. The fact that the ratios of the various Yukawa couplings do not enter Eqs. (109) - (115) implies that these results are valid for any $\tan \beta$ regime.

One can also obtain the complete analytic solutions of the RG equations for the trilinear supersymmetry breaking parameters $A_{i}$ in an analogous manner. The expressions for these are lengthy, and will not be written here. Since the solutions for the quasi-fixed points must be obtained either iteratively from (109), or numerically from the RG equations, we shall instead study the numerical solutions for the quasi-fixed points for these $A$ parameters, as well as the Yukawa couplings, in the following.

\section{A. Quasi-fixed points for the Yukawa couplings}

In order to determine the quasi-fixed points, one can solve the RG equations for the various couplings numerically. We shall do so in the next section. However, before doing so it is instructive and useful to obtain an analytical estimate of the quasi-fixed point values for these couplings. As stated above, we shall study the quasi-fixed point for the couplings $h_{t}, h_{b}, h_{\tau}, \lambda, \kappa, \lambda_{233}^{\prime \prime}$, since only the fixed point (54) corresponding to these couplings is stable.

Writing the RG equations for (14) - (18), and (21) for these couplings in terms of $\tilde{Y}^{\prime} s$ defined in (94) - (96), the existence of quasi-fixed point requires [21]

$$
\frac{d \tilde{Y}_{t}}{d t} \simeq \frac{d \tilde{Y}_{b}}{d t} \simeq \frac{d \tilde{Y}_{\tau}}{d t} \simeq \frac{d \tilde{Y}_{\lambda}}{d t} \simeq \frac{d \tilde{Y}_{\kappa}}{d t} \simeq \frac{d \tilde{Y}^{\prime \prime}}{d t} \simeq 0
$$

The RG equations for $Y_{\lambda}$ and $\tilde{Y}_{\kappa}$ lead to 


$$
\tilde{Y}_{\lambda}=\tilde{Y}_{\kappa}=0
$$

thereby implying that the quasi-fixed point values for $\lambda$ and $\kappa$ are zero. Substituting these values in the remaining $R G$ equations, we get

$$
\tilde{Y}_{\tau}^{*}=\frac{3}{730}\left[121 \tilde{\alpha}_{1}+100\left(\tilde{\alpha}_{2}-\tilde{\alpha}_{3}\right)\right] \simeq-0.0016,
$$

where we have used $\alpha_{1} \simeq 0.017, \alpha_{2} \simeq 0.033, \alpha_{3} \simeq 0.1$ at the effective supersymmetry scale, which we take to be $1 \mathrm{TeV}$. This solution is clearly unphysical. We are, therefore, led to set $\tilde{Y}_{\tau}^{*}=0$, yielding the solution

$$
\begin{gathered}
\tilde{Y}_{t}^{*}=\frac{47 \tilde{\alpha}_{1}+225 \tilde{\alpha}_{2}+200 \tilde{\alpha}_{3}}{425} \simeq 0.0052, \\
\tilde{Y}_{b}^{*}=\frac{13 \tilde{\alpha}_{1}+225 \tilde{\alpha}_{2}+200 \tilde{\alpha}_{3}}{425} \simeq 0.0052, \\
\tilde{Y}^{\prime \prime *}=\frac{2\left(11 \tilde{\alpha}_{1}-45 \tilde{\alpha}_{2}+130 \tilde{\alpha}_{3}\right)}{255} \simeq 0.0073,
\end{gathered}
$$

which leads to the quasi-fixed point values for these couplings

$$
\begin{aligned}
h_{t}^{*} & \simeq 0.91 \\
h_{b}^{*} & \simeq 0.90 \\
\lambda_{233}^{\prime \prime} & \simeq 1.08
\end{aligned}
$$

We note that these quasi-fixed point values for the respective couplings are similar to the values obtained for them in MSSM [11].

\section{B. Quasi-fixed points for trilinear soft supersymmetry breaking parameters}

We now turn our attention to the renormalization group equations (23) - (31) for the $A$ parameters, and their quasi-fixed points. Since the quasi-fixed point values for $h_{\tau}, \lambda, \kappa$ are trivial, we cannot determine the quasi-fixed point values for $A_{\tau}, A_{\lambda}$, and $A_{\kappa}$, and, therefore, ignore the coresponding renormalization group equations (25) - (27). In rest of the $A$ equations, we substitute $h_{\tau}=\lambda=\kappa=0$, and obtain the equations that the remaining $A$ parameters must satisfy in order for us to determine the quasi-fixed point solution:

$$
\begin{array}{r}
6 \tilde{Y}_{t} A_{t}+\tilde{Y}_{b} A_{b}+2 \tilde{Y}^{\prime \prime} A_{\lambda^{\prime \prime}}-\frac{16}{3} \tilde{\alpha}_{3} M_{3}-3 \tilde{\alpha}_{2} M_{2}-\frac{13}{15} \tilde{\alpha}_{1}=0 \\
\tilde{Y}_{t} A_{t}+6 \tilde{Y}_{b} A_{b}+2 \tilde{Y}^{\prime \prime} A_{\lambda^{\prime \prime}}-\frac{16}{3} \tilde{\alpha}_{3} M_{3}-3 \tilde{\alpha}_{2} M_{2}-\frac{7}{15} \tilde{\alpha}_{1}=0 \\
2 \tilde{Y}_{t} A_{t}+2 \tilde{Y}_{b} A_{b}+6 \tilde{Y}^{\prime \prime} A_{\lambda^{\prime \prime}}-8 \tilde{\alpha}_{3} M_{3}-\frac{4}{5} \tilde{\alpha}_{1}=0
\end{array}
$$

These yield the following quasi-fixed point solution: 


$$
\begin{gathered}
A_{t}^{*}=\frac{47 \tilde{\alpha}_{1} M_{1}+225 \tilde{\alpha}_{2} M_{2}+200 \tilde{\alpha}_{3} M_{3}}{425 \tilde{Y}_{t}^{*}} \simeq 0.77 m_{\tilde{g}}, \\
A_{b}^{*}=\frac{13 \alpha_{1} M_{1}+225 \tilde{\alpha}_{2} M_{2}+200 \tilde{\alpha}_{3} M_{3}}{425 \tilde{Y}_{b}^{*}} \simeq 0.78 m_{\tilde{g}}, \\
A_{\lambda^{\prime \prime}}^{*}=\frac{2\left(11 i \tilde{\alpha}_{1} M_{1}-45 \tilde{\alpha}_{2} M_{2}+130 \tilde{\alpha}_{3} M_{3}\right)}{255 \tilde{Y}^{\prime \prime *}} \simeq 1.02 m_{\tilde{g}},
\end{gathered}
$$

where $m_{\tilde{g}}$ is the gluino mass $\left(=M_{3}\right)$ defined at the weak scale. For the numerical estimates in (128) - (130), we have used the fact that the gaugino masses scale as the square of the gauge couplings, and that $\alpha_{G} \simeq 0.041$ at the grand unified scale $M_{G} \simeq 10^{16} \mathrm{GeV}$. One must compare these quasi-fixed point values with the true fixed-point values (93). Since the quasi-fixed points for the Yukawa couplings represent their upper bounds, the quasi-fixed point values (128) - (130) provide a lower bound on the corresponding $A$ parameters. We note that inspite of the presence of additional trilinear couplings in the superpotential of the NMSSM, the quasi-fixed point behaviour is similar to the corresponding quasi-fixed point behaviour in MSSM with baryon and lepton number violation.

\section{NUMERICAL RESULTS AND DISCUSSION}

In the previous section we have obtained the approximate quasi-fixed point values for the Yukawa couplings and the $A$ parameters by an algebraic solution of the corresponding RG equations. The RG equations are a set of coupled first-order differential equations that must be solved numerically to obtain accurate values for the fixed points. We have numerically solved the RG equations for the Yukawa couplings, and the $A$ parameters. We now present the results of such a numerical analysis.

In Fig.1 we show the fixed point behaviour of the top-quark Yukawa coupling as a function of the logarithm of the scale parameter $\mu$. We have included the evolution equations for the b-quark, the $\tau$-lepton Yukawa coupling, and the trilinear couplings $\lambda$ and $\kappa$, as well as the B-violating coupling $\lambda_{233}^{\prime \prime}$, and evolved them from the initial value $\ln \left(\mu / M_{G}\right)=0$ to the final value $\ln \left(\mu / M_{G}\right) \simeq-33$ at the weak scale, for the numerical solution. It is seen that for all $h_{t} \gtrsim 1$ at the GUT scale, the top-quark Yukawa coupling approaches its quasi-fixed point at the weak scale. We note that the numerical evolution of fixed point approaches but does not exactly reproduce the approximate analytical value in (122). In Figs. 2 and 3 we present the corresponding approach to the quasi-infrared fixed point for the couplings $h_{b}$ and $\lambda_{233}^{\prime \prime}$, respectively. These infrared fixed points provide a model independent theoretical upper bound on the $B$-violating coupling $\lambda_{233}^{\prime \prime}$. It is worthwhile to point out here that the fixed point value for $\lambda_{233}^{\prime \prime} \simeq 1.08$ is more than a factor of 3 lower than what would be a naive perturbative upper bound of $\lambda_{233}^{\prime \prime} \lesssim \sqrt{4} \pi \simeq 3.5$ for this coupling.

In Figs. 4, 5 and 6, we present the fixed point behaviour of the corresponding $A$ parameters. We notice the remarkable focussing property seen in the fixed point behaviour of all the $A$ parameters. Again, we notice that the numerical evolution of the fixed point approaches, but does not actually reproduce, the approximate analytical values of Eqs. (128), (129), and (130). Since the quasi-fixed point value for the $A$ parameter is inversely proportional to the quasi-fixed point value of the Yukawa coupling, it provides a lower bound on the corresponding $A$ parameter. 


\section{SUMMARY AND CONCLUSIONS}

We have carried out a comprehensive study of the renormalization group flow in the nonminimal supersymmetric standard model with all the third generation Yukawa couplings, and the trilinear couplings $\lambda$ and $\kappa$, and with highest generation baryon and lepton number violation. We have shown that the simultaneous fixed point for the top- and bottom-Yukawa couplings, and the $B$-violating coupling $\lambda_{233}^{\prime \prime}$, is the only fixed point that is stable in the infrared region. However, the top-quark mass predicted by this fixed point is incompatible with measured value of the top mass. This fixed point, therefore, provides a process-independent lower bound on the baryon number violating coupling $\lambda_{233}^{\prime \prime}$ at the electroweak scale.

We have shown that all other possible fixed point solutions are either unphysical, or unstable, in the infra-red region. In particular there is no infrared fixed point with simultaneous $B$ and $L$ violation. This result is analogous to that found in MSSM with $B$ and $L$ violation [11].

We have also carried out the renormalization group analysis of the corresponding trilinear soft supersymmetry breaking parameters. We have obtained the true fixed points for these parameters, which serve as upper bounds on these parameters.

Since the true fixed points are not reached in practice at the electroweak scale, we have also obtained the quasi-fixed points of the Yukawa couplings and the trilinear parameters. The quasi-fixed point values for the Yukawa couplings are numerically very close to the values obtained previously by ignoring the $\tau$ Yukawa coupling. Since the quasi-fixed points are reached for large initial values of the couplings at the GUT scale, these reflect on the assumption of perturbative unitarity, or the absence of Landau poles, of the corresponding couplings. These quasi-fixed points, therefore, provide an upper bound on the relevant Yukawa coupling, especially the baryon number violating coupling $\lambda_{233}^{\prime \prime}$. From the true fixed point and the quasi-fixed point ananlysis we are able to constrain the baryon number violating coupling $0.97 \lesssim \lambda_{233}^{\prime \prime} \lesssim 1.08$ in a model independent manner. We would like to emphasize that this infrared fixed point upper bound on $\lambda_{233}^{\prime \prime}$ is more than a factor of 3 lower than the naive perturbative upper bound on this coupling, and represents a significant constraint on it. We have complemented the quasi-fixed point analysis of the Yukawa couplings with an analysis of the corresponding soft supersymmetry breaking trilinear couplings. We have shown that the $A$ parameters for the top- and bottom-quark Yukawa couplings, and the baryon number violating couplings all show striking convergence properties. This strong focussing property is quite independent of the input parameters at the unification scale (or equivalently the pattern of supersymmetry breaking), and the $A$ parameters are, therefore, fully determined in the quasi-fixed regime. In particular, we have constrained

the $A$ parameters to be $0.77 \lesssim A_{t} / m_{\tilde{g}} \lesssim 1,0.78 \lesssim A_{b} / m_{\tilde{g}} \lesssim 1$, and $A_{\lambda^{\prime \prime}} / m_{\tilde{g}} \simeq 1$. These constraints are analogous to those found in MSSM for these parameters. Thus, the infrared fixed point behaviour of NMSSM with $B$ and $L$ violation is similar to that of MSSM, and may be universal for supersymmetric models based on $S U(2)_{L} \times U(1)_{Y}$ gauge group. 


\section{ACKNOWLEDGEMENTS}

The author is supported by the University Grants Commission under project No. 3063/98/SA-III. He would like to thank the Theory Group at DESY for its hospitality while this work was completed. 


\section{REFERENCES}

[1] J. Wess and J. Bagger, "Supersymmetry and Supergravity" (Princeton University Press, Princeton, NJ, 1992); P. Nath, R. Arnowitt and A. H. Chamseddine, "Applied N = 1 Supergravity" (World Scientific, Singapore, 1984).

[2] For a review and references, see e.g., B. Schrempp and M. Wimmer, Prog. Part. Nucl. Phys. 37, 1 (1996).

[3] B. Pendleton and G. G. Ross, Phys. Lett. B98, 291 (1981); M. Lanzagorta and G. G. Ross, Phys. Lett. B349, 319 (1995).

[4] C. T. Hill, Phys. Rev. D24, 691 (1981).

[5] B. C. Allanach and S. F. King, Phys. Lett. B407, 124 (1997).

[6] S. A. Abel and B. C. Allanach, Phys. Lett. B415, 371 (1997).

[7] I. Jack and D. R. T. Jones, Phys. Lett. B443, 177 (1998).

[8] S. Weinberg, Phys. Rev. D26, 287 (1982); N. Sakai and T. Yanagida, Nucl. Phys. B197, 133 (1982).

[9] G. Farrar and P. Fayet, Phys. Lett. B76, 575 (1978).

[10] B. Ananthanarayan and P. N. Pandita, Phys. Lett. B454, 84 (1999).

[11] B. Ananthanarayan and P. N. Pandita, Phys. Rev D62, 036009 (2000), and references therein; B. Ananthanarayan and P. N. Pandita, Phys. Rev. D62, 0760XX (2001) (in press).

[12] P. Fayet, Nucl. Phys. B90, 104 (1975); R. K. Kaul and P. Majumdar, Nucl. Phys. B199, 36 (1982); J. Ellis et al., Phys. Rev. D39, 844 (1989); P. N. Pandita, Phys. Lett. B318, 338 (1993); Z. Phys. C59, 575 (1993).

[13] P. N. Pandita and P. Francis Paulraj, Phys. Lett. B462, 294 (1999).

[14] See, e.g., R. Barbier et al., hep-ph/9810232 (unpublished); B. Allanach et al., hepph/9906224.

[15] N. Falck, Z. Phys. C30, 247 (1986); S.P. Martin and M.T. Vaughn, Phys. Rev. D50, 2282 (1994).

[16] These equations are generalizations of the RG equations in P.N. Pandita and P. Francis Paulraj, Phys. Lett. B462, 294 (1999) when we consider simultaneous highest generation baryon and lepton number violating couplings in NMSSM.

[17] S. Codoban and D. I. Kazakov, Eur. Phys. J. C13, 671 (2000).

[18] B. Gato et al., Nucl. Phys. B253, 285 (1985); N. K. Falck, Z. Phys. C30, 247 (1986); S. P. Martin and M. T. Vaughn, Phys. Rev. D50, 2282 (1994).

[19] Particle Data Group, D. E. Groom et al., Eur. Phys. J. C, 1 (2000).

[20] We follow here the notation of B. Ananthanarayan and P. N. Pandita, Phys. Rev. D62, 0760XX (2001) (in press). See also G. Auberson and G. Moultaka, Eur. Phys. J. C12, 331 (2000); D. Kazakov and G. Moultaka, Nucl. Phys. B577, 121 (2000).

[21] V. Barger, M. S. Berger, R. J. N. Phillips, and T. Wöhrmann, Phys. Rev. D53, 6407 (1996) 


\section{Figure Captions}

Fig. 1. The quasi-fixed point of the renormalization group evolution of the top-quark Yukawa coupling $h_{t}$ as a function of the logarithm of the energy scale. We have taken the initial values of $h_{t}$ at the grand unified scale $M_{G} \sim 10^{16}$ to be 4.0, 3.0, 2.0, and 1.0, and evolved them down to $\ln \left(\mu / M_{G}\right) \simeq-33$. The initial values of other Yukawa couplings are $h_{b}=1.0, h_{\tau}=0, \lambda=\kappa=0.5$ and $\lambda_{233}^{\prime \prime}=2.0$.

Fig. 2. The renormalization group evolution of the bottom-quark Yukawa coupling $h_{b}$ as a function of the logarithm of the energy scale. The initial values of $h_{b}$ at $M_{G}$ are 4.0, 3.0, 2.0, and 1.0. The initial values of other Yukawa couplings are $h_{t}=1.0, h_{\tau}=0, \lambda=$ $\kappa=0.5, \lambda_{233}^{\prime \prime}=2.0$.

Fig. 3. Th equasi-fixed point behaviour of the baryon number violating Yukawa coupling $\lambda_{233}^{\prime \prime}$ as a function of the logarithm of the energy scale. The initial values are $\lambda_{233}^{\prime \prime}=4.0,3.0,2.0,1.0$. The initial values of other other parameters are are $h_{t}=h_{b}=$ $1.0, h_{\tau}=0, \lambda=\kappa=0.5$.

Fig. 4. Renormalization group evolution of ratio $A_{t} / m_{\tilde{g}}$ as a function of the logarithm of the energy scale for several different initial values at the grand unified scale $M_{G}$. The initial values at $M_{G}$ are $-4,-3,-2,-1,0,1,2,3,4$. The initial values for other parameters at $M_{G}$ are $h_{t}=4.0, h_{b}=1.0, h_{\tau}=0, \lambda=\kappa=0.1, \lambda_{233}^{\prime \prime}=1.0$, and $A_{b} / m_{\tilde{g}}=2.0, A_{\lambda_{233}^{\prime \prime}} / m_{\tilde{g}}=3.0$.

Fig. 5. Renormalization group evolution of the ratio $A_{b} / m_{\tilde{g}}$ as a function of the logarithm of the energy scale. The initial values of the ratio are $-4,-3,-2,-1,0,1,2,3,4$. Other parameters at the scale $M_{G}$ are $h_{t}=1.0, h_{b}=4.0, h_{\tau}=0, \lambda=\kappa=0.1, \lambda_{233}^{\prime \prime}=1.0$, and $A_{t} / m_{\tilde{g}}=2.0, A_{\lambda_{233}^{\prime \prime}} / m_{\tilde{g}}=2.57$.

Fig. 6. Renormalization group evolution of the trilinear coupling $A_{\lambda_{23}^{\prime \prime}} / m_{\tilde{g}}$. The initial values of the ratio are $-4,-3,-2,-1,0,1,2,3,4$. Other parameters at the scale $M_{G}$ are $h_{t}=h_{b}=1.0, h_{\tau}=0, \lambda=\kappa=0.1$, and $A_{t} / m_{\tilde{g}}=2.0 A_{b} / m_{\tilde{g}}=2.0$. 


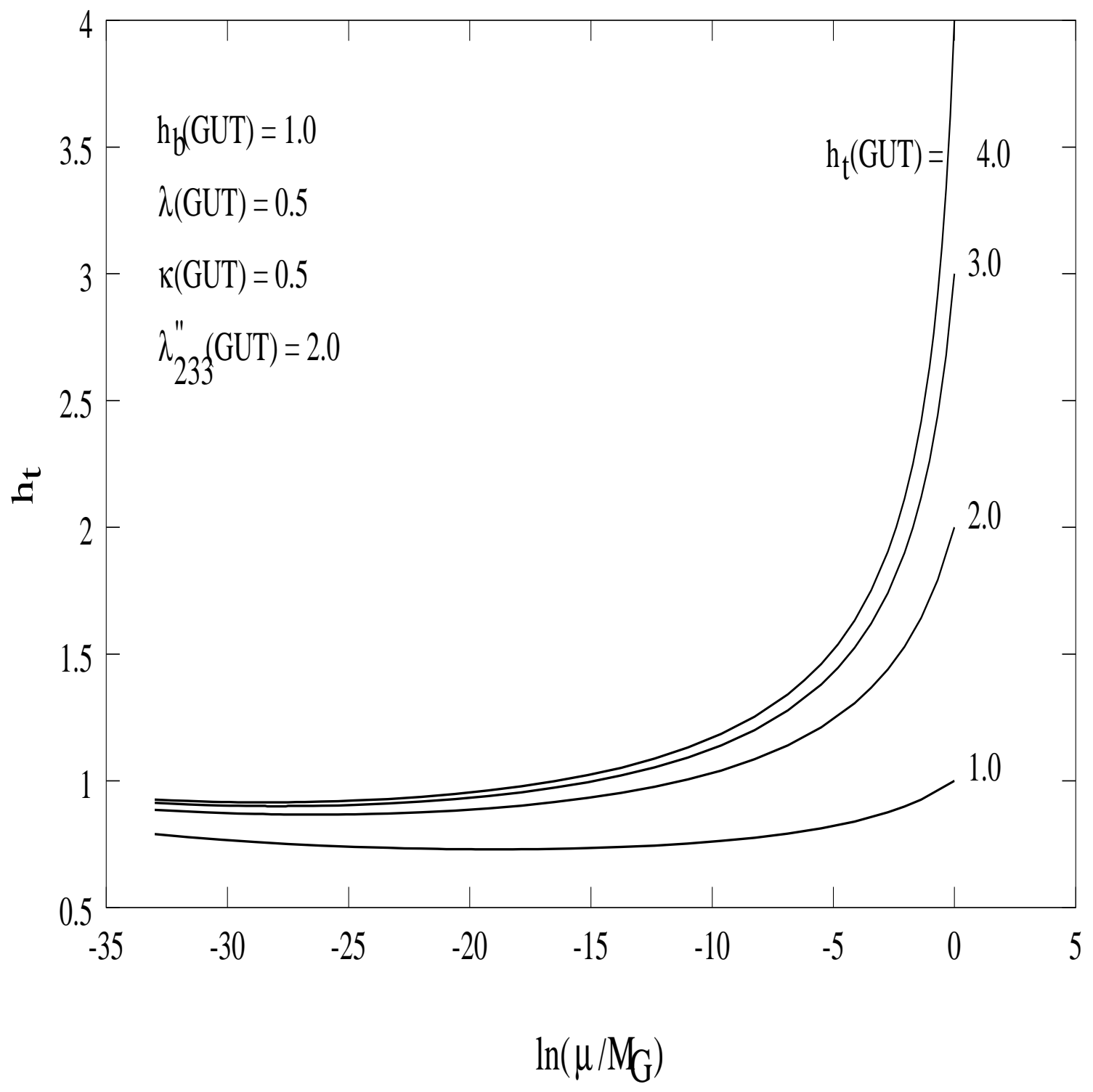

FIG. 1. 


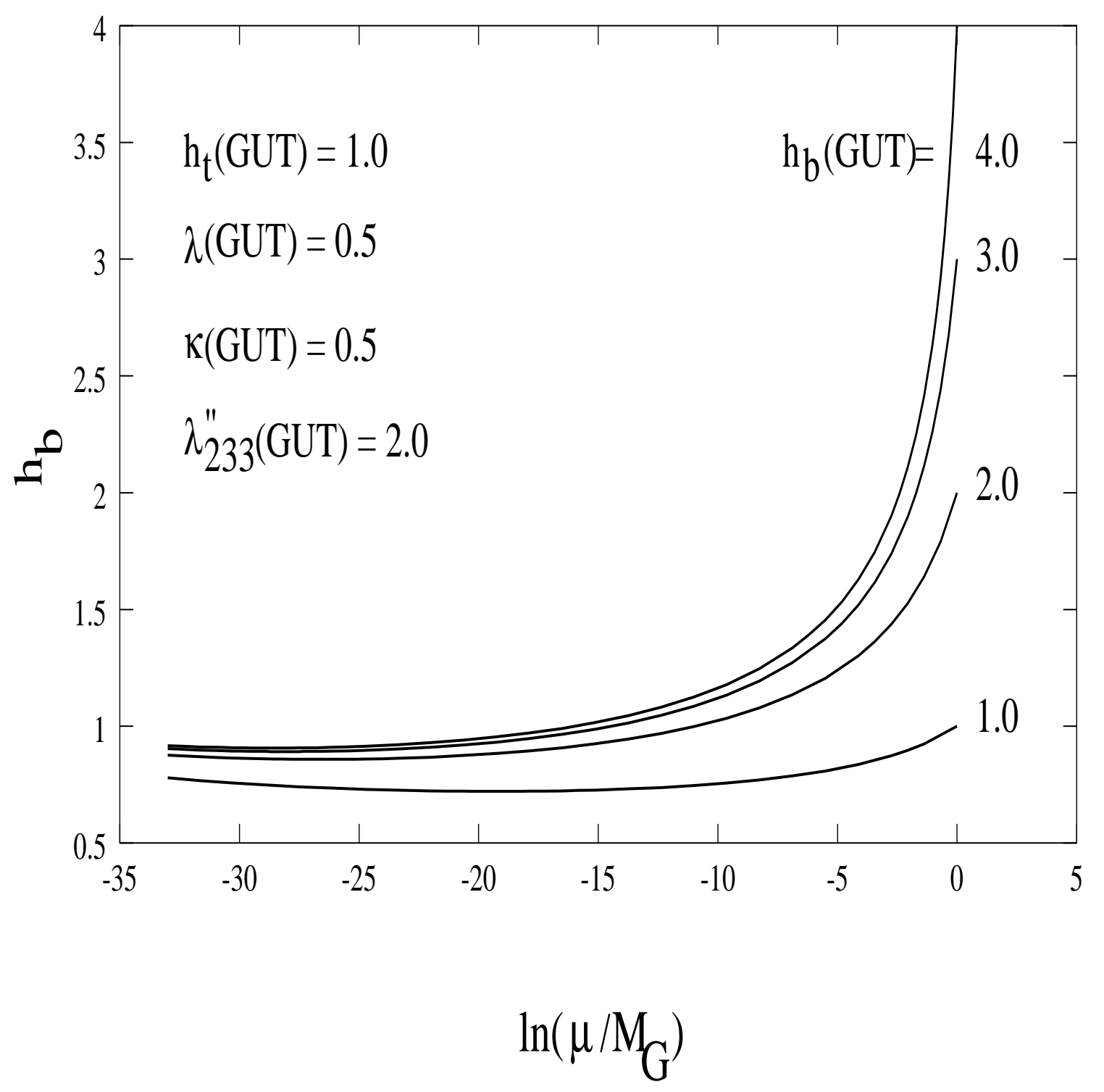

FIG. 2. 


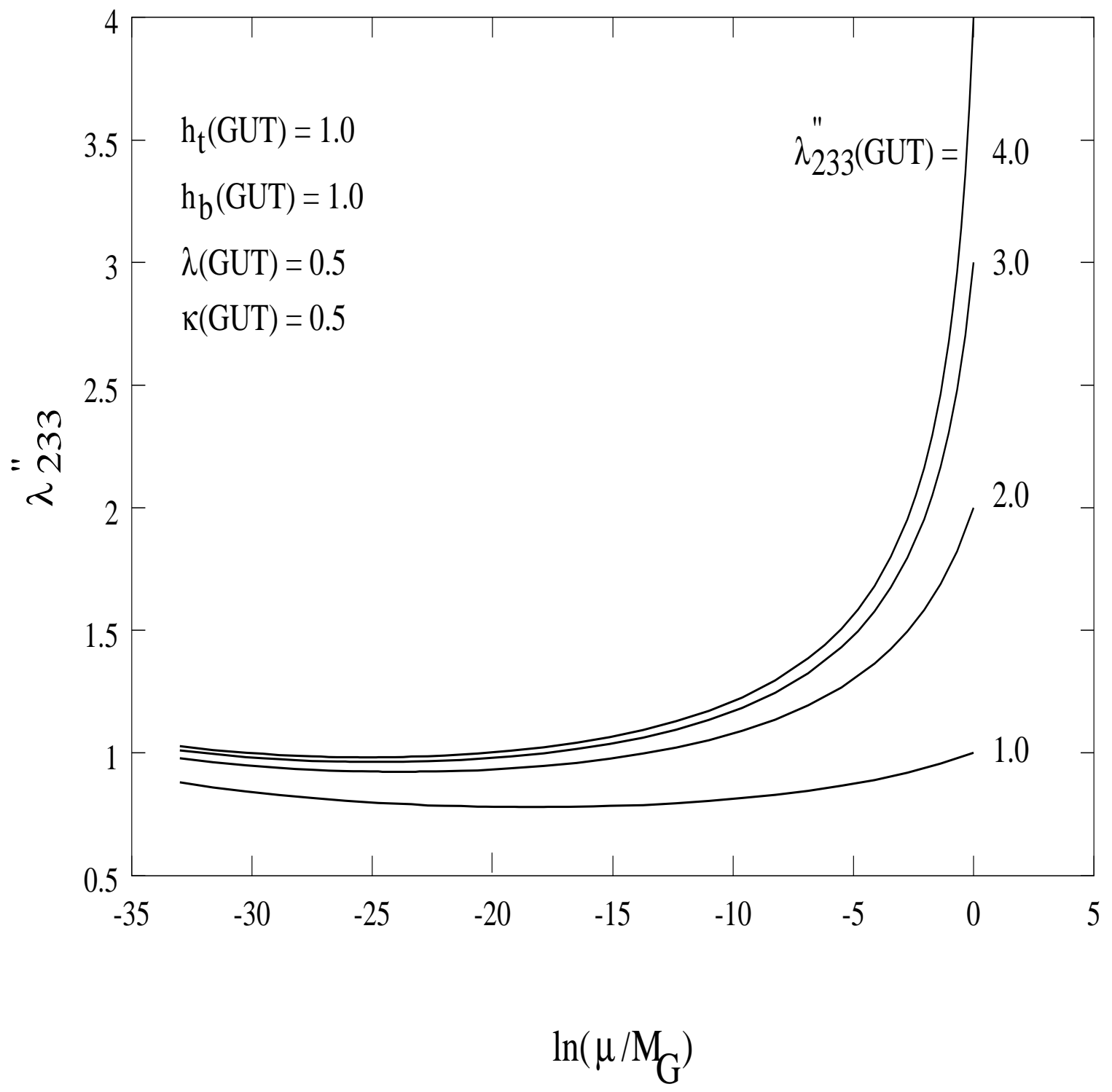

FIG. 3. 


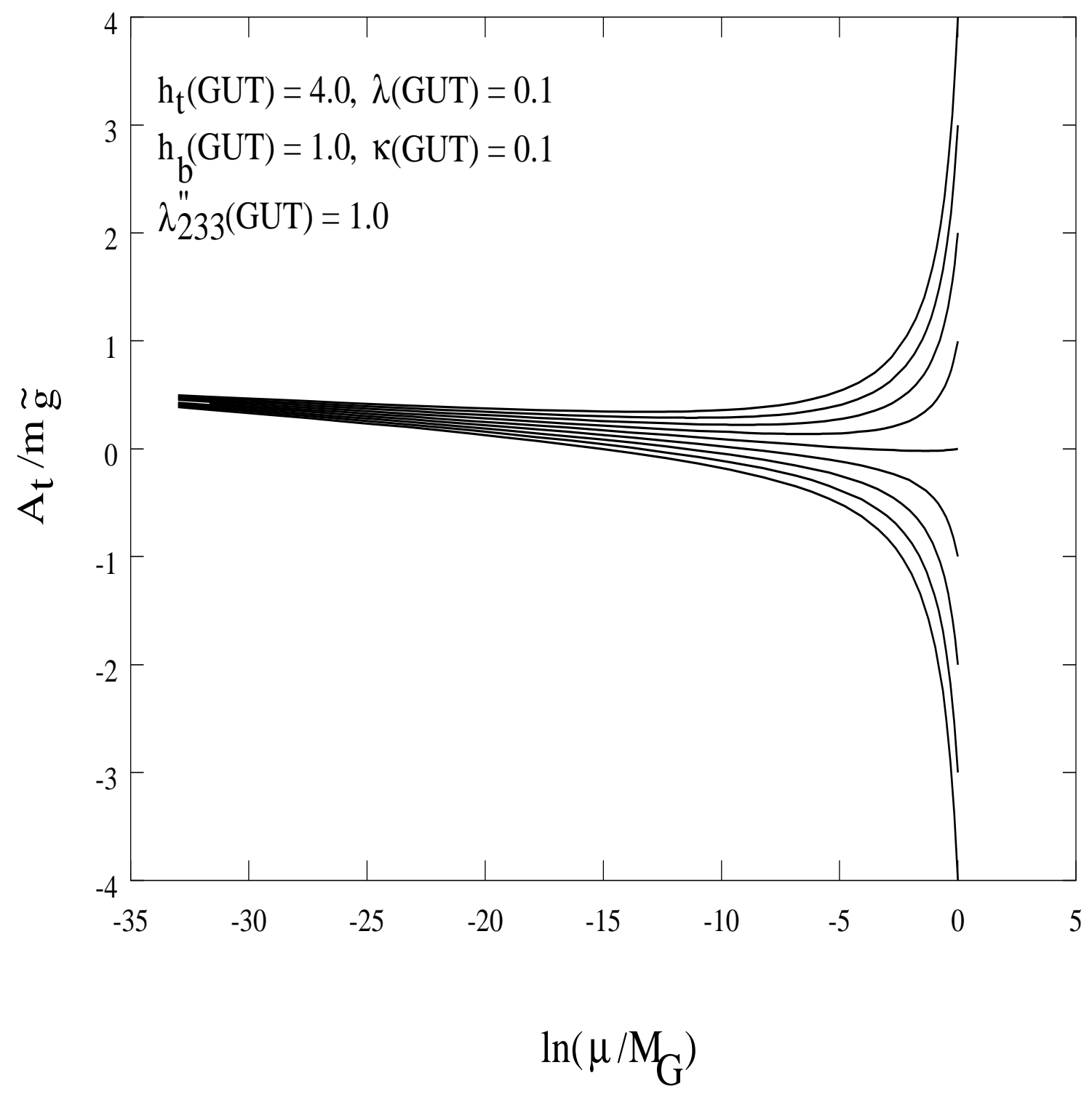

FIG. 4. 


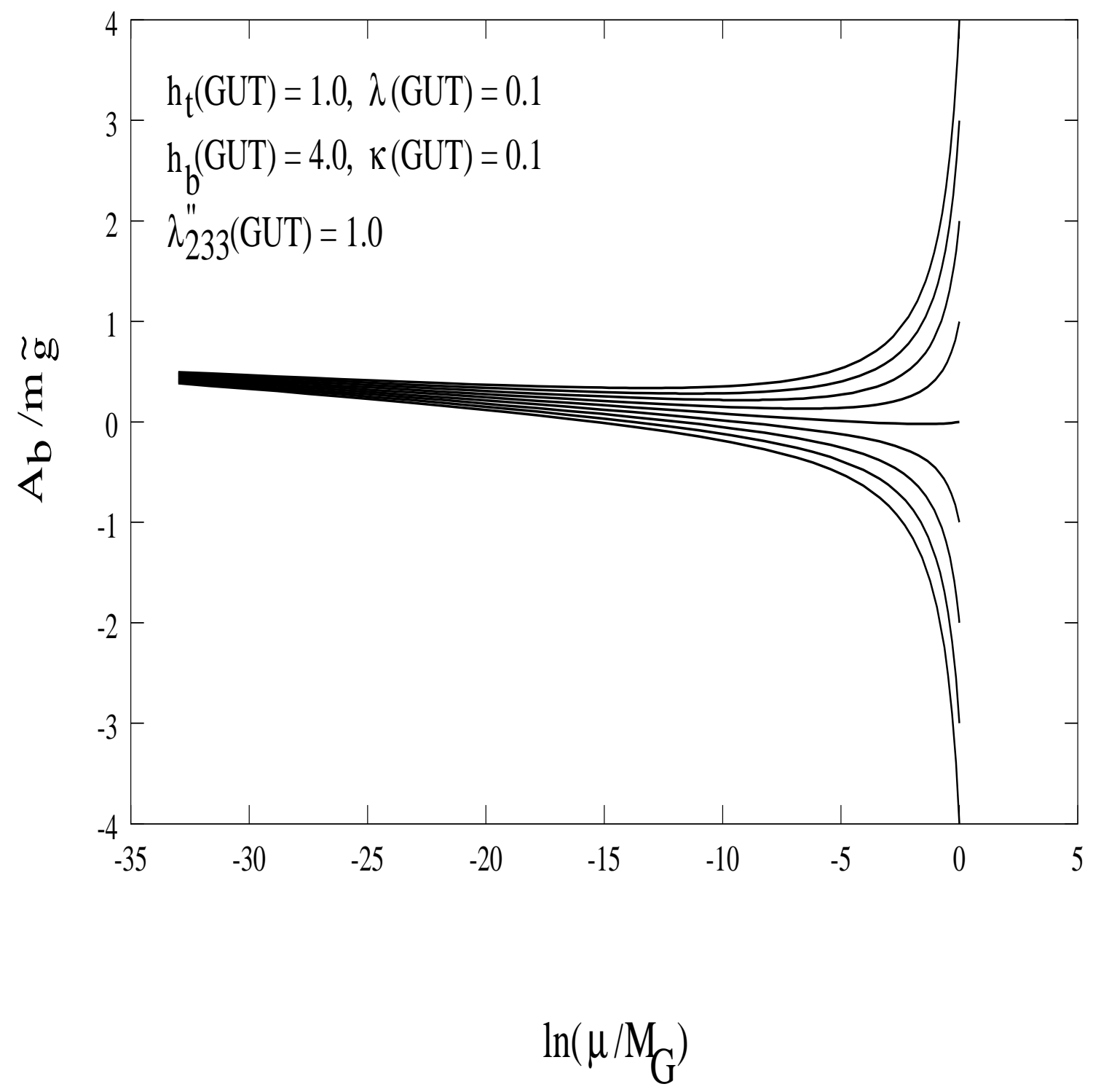

FIG. 5. 


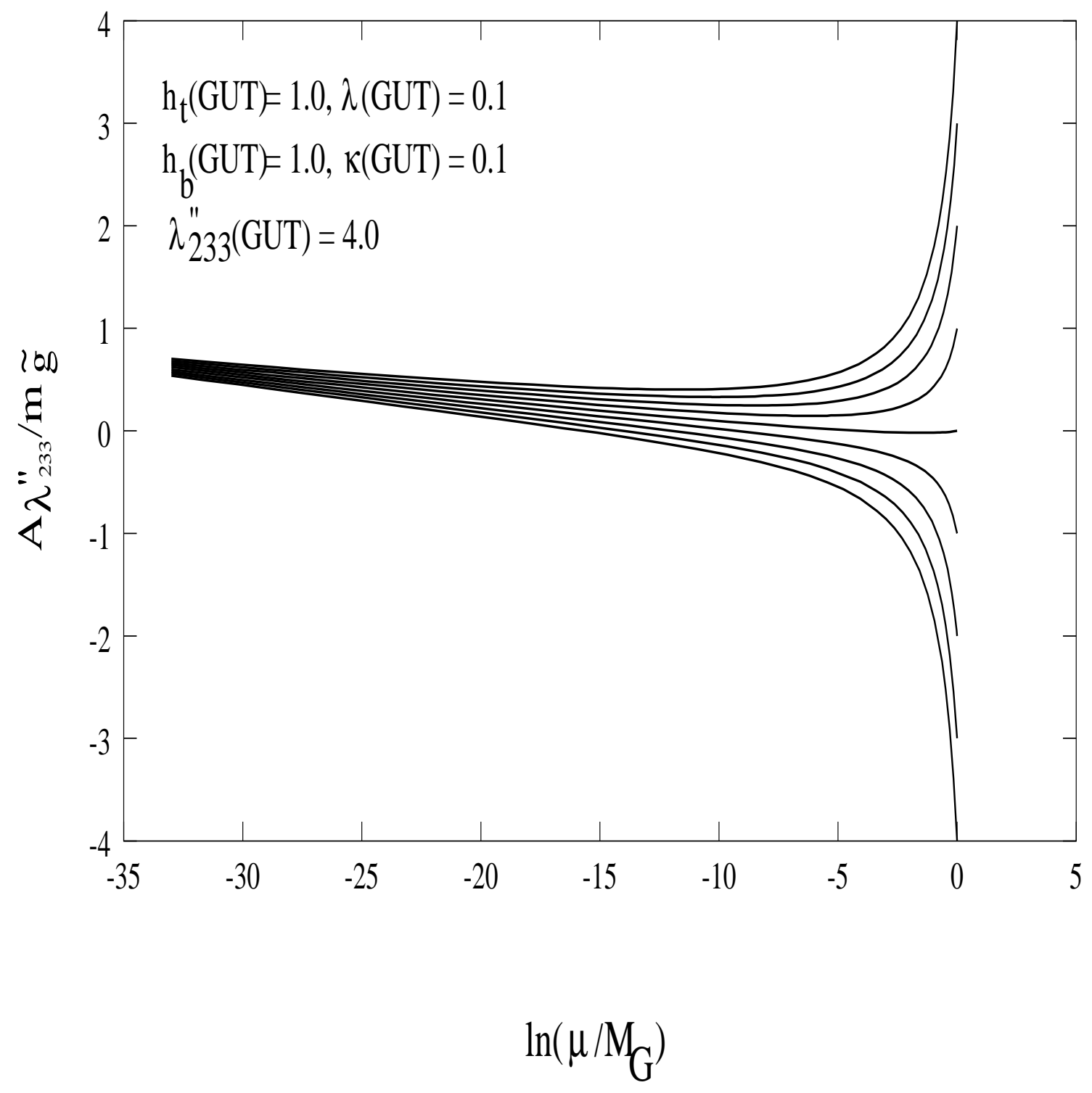

FIG. 6. 